\title{
Measurement of retinal vessel widths from fundus images based on 2D modelling
}

\author{
James Lowell Andrew Hunter David Steel Ansu Basu Robert Ryder \\ R. Lee Kennedy
}

J. Lowell and A. Hunter, Dept. Applied Computer Science, University of Lincoln, Brayford Pools, Lincoln, UK. ahunter@lincoln.ac.uk

D. Steel, Sunderland Eye Infirmary, Queen Alexandra Road, Sunderland, SR2 9HP

A. Basu and R. Ryder, Dept. Diabetes and Endocrinology, City Hospital, Dudley Rd., Birmingham, B18 7QH, UK

R.L. Kennedy, Diabetes Unit, Queen's Medical Centre, University Hospital Nottingham, NG7 2UH. 


\begin{abstract}
Changes in retinal vessel diameter are an important sign of diseases such as hypertension, arteriosclerosis and diabetes mellitus. Obtaining precise measurements of vascular widths is a critical and demanding process in automated retinal image analysis as the typical vessel is only a few pixels wide. This paper presents an algorithm to measure the vessel diameter to sub-pixel accuracy. The diameter measurement is based on a two-dimensional difference of Gaussian model, which is optimized to fit a two-dimensional intensity vessel segment. The performance of the method is evaluated against Brinchmann-Hansen's half height, Gregson's rectangular profile and Zhou's Gaussian model. Results from 100 sample profiles show that the presented algorithm is over $30 \%$ more precise than the compared techniques and is accurate to a third of a pixel.
\end{abstract}

\title{
I. INTRODUCTION
}

This paper presents an algorithm for the automatic measurement of blood vessel diameters in retinal images. This paper focuses on the specific technical issue of accurate diameter measurement, and does not address the other stages involved in the diagnosis of disease based on vascular pathology. In particular, we assume that approximate vessel center lines have been previously found. We argue that the diameter measurement stage is sufficiently critical to deserve careful study in its own right. Diameter measurements are made by fitting a 2D model, which resembles an idealized cross sectional profile running along the length of a vessel segment in a small region of interest. The model is fitted on an intensity image produced by extracting the green channel from an original color digital image. We evaluate the algorithm against alternative approaches using a set of 100 cross section profiles from five down-sampled high definition fundus images, and present results showing that it is significantly superior to previously published methods.

\section{A. Motivation}

The retinal vessels are the only part of the central circulation that can be viewed directly and studied in detail [1]. Segmentation and measurement of the retinal vessels is therefore of central interest in a number of diseases, including some that are primarily retinal disorders, such as diabetic retinopathy - the leading cause of blindness in the working population of industrialized countries [2] - and others, such as arteriosclerosis, hypertension and stroke, which are not primarily retinal, but where it is known that changes in the morphology of the retinal vessels do occur, and can be predictive of risk. In general, automated segmentation and measurement 
is desirable to improve reliability and reproducibility of measurements, and in some cases to support wide-spread automated screening for vascular conditions.

A variety of morphological changes occur to retinal vessels in different disease conditions; however, in this paper we are exclusively concerned with changes in the calibre (diameter, width) of vessels. The change in width of retinal vessels within the fundus is believed to be indicative of the risk level of diabetic retinopathy [3]; venous beading (unusual variations in diameter along a vein) is one of the most powerful predictor of proliferate diabetic retinopathy [2]. Generalized and focal retinal arteriolar narrowing and arteriovenous nicking have been shown to be strongly associated with current and past hypertension reflecting the transient and persistent structural effects of elevated blood pressure on the retinal vascular network [4] [5]. In addition retinal arteriolar bifurcation diameter exponents have been shown to be changed significantly in patients with peripheral vascular disease and arteriosclerosis [6] and a variety of retinal microvascular abnormalities have been shown to be related to the risk of stroke [7]. Future precise retinal vascular analysis may allow risk stratification of a variety of arteriosclerotic diseases and clarify the microvascular contributions to clinical cardiovascular disease. It is worth noting that, in all these cases, it is not the absolute diameter of the vessel that is of interest, but variation in the diameter along a vessel.

Segmentation of vessels and measurement of the vascular diameter are two critical and challenging technical tasks in any system attempting automated diagnosis of vascular conditions. A number of methods for segmenting the vascular network have been reported in the literature [8] [9], with some success at least for larger vessels, although many papers gloss over the difficulty of reliably segmenting vessels in images of diseased retina, where there are significant distractors present; robust segmentation therefore remains an important research issue.

This paper concentrates on the diameter measurement issue. On a standard $30^{\circ}$ fundal image with resolution $760 \times 570$, moderately sized vessels are as little as 6-8 pixels in width. Consequently, even with improving fundal camera resolutions, errors in diameter measurement of the order of a single pixel are large enough to significantly compromise attempts to characterize anomalies in vessel width, except for gross anomalies on the largest vessels. We are not aware of any studies indicating exactly at what point vascular diameter changes become clinically significant in different conditions - it may indeed be that such studies have not yet been conducted 
due to the difficulty in making sufficiently accurate measurements. Nonetheless, given the level of accuracy of existing algorithms and the fact that vascular conditions are progressive, we believe that improvements in the accuracy of the diameter measurement algorithm may potentially prove very valuable.

This paper introduces a new algorithm, based on fitting a local 2D vessel model, which can measure vascular width to an accuracy of about one third of a pixel. We demonstrate that this is significantly better performance than previously published methods. The method may be integrated with any vessel segmentation algorithm [8] [9], and with any algorithm to diagnose pathology on the basis of vessel calibre. Although we do not, in this paper, attempt to apply the diameter measurement algorithm in diagnosis of any particular vascular pathology, we believe the technique is of sufficiently widespread potential to be of interest in its own right.

\section{B. Contents of Paper}

In section II we outline the retinal vessel structure and discuss the true versus apparent width of the blood column. We briefly describe common vessel segmentation algorithms in Section III, and overview previously published diameter-measuring algorithms in Section IV. In section $\mathrm{V}$ we discuss the new diameter-measurement algorithm. We evaluate the algorithm against alternatives from the literature in section VI and conclude in Section VII.

\section{Retinal Vessel Structure}

Light emitted from a fundus camera or ophthalmoscopy is reflected back by the retina and choroids. Blood vessels absorb some of the reflected light, absorbing into the wall and the blood. When an image of a blood vessel is viewed from point $\mathrm{C}$ (see figure 1), the apparent width of the vessel is believed to be the width of the streaming column of erythrocytes viewed perpendicular to the flow direction (point P) [10]. The surrounding plasma zone and the vessel wall are transparent. Variations in the thickness of the vessel wall and the index of refraction have negligible influence on the apparent width of the blood column.

Due to the measured indices of refraction in the plasma and vitreous, it is accepted that within wide physiological limits the apparent width of the blood column is proportional to the true width [10]. Looking at the retinal blood vessel as a vascular cross-section, the overall transmittance through a vessel including the blood column and wall resembles a Gaussian curve. Finding 


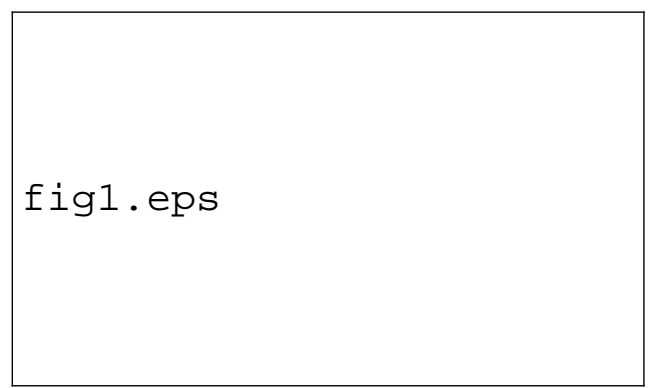

Fig. 1. The apparent and true width of the blood column Based on Brinchmann-Hansen, 0; Heier, H. Acta Ophthalmolog. Suppl. 1986,179,29-32.

the point that the blood column meets the wall on that curve is extremely difficult and has inspired many algorithms (see section IV). To complicate the problem further, some blood vessels include a light streak known as a light reflex that run down the central length of the blood vessel. Naïve approaches can be fooled into thinking that the light reflex is the edge of the vessel.

The light reflex is understood to run across the surface of the plasma zone and the blood column and is believed to be generated from a rough reflecting surface and the intravascular column of erythrocytes [10]. Light reflexes are more common in younger retinas due to their increased reflective surfaces. However, arteriosclerosis can also affect the appearance of the light reflex by changing the reflective index of the vessel wall, thus increasing the observed intensity.

\section{OVERVIEW OF VESSEL SEGMENTATION ALGORITHMS}

This paper presents an algorithm for measuring the vascular diameter, which is designed for use in a larger system. The overall process consists of detecting and segmenting vessels, sampling points along vessels; measuring diameters; and drawing conclusions about vascular health. The contribution of this paper is in vascular measurement, not segmentation. Nonetheless, in this section we briefly discuss the first of these stages, to place the diameter measurement method in context.

In the vascular segmentation stage, the approximate centreline of vessels is detected. A number of algorithms for this process have been published. The most popular approach is to use an elongated linear filter with a Gaussian or similar profile, rotated through a number of angles and convolved with the image [8] [9] [11] [12]. The highest response filter is selected for each pixel and can be thresholded to provide a vessel image, which is subsequently thinned and cleaned up 
to identify vessel segments. This convolution approach encounters a number of problems due to the retinal background variation and low contrast of the fainter vessels with the local surroundings. It is also prone to false responses around bright objects such as exudates and reflection artifacts.

In this paper we use our own non-linear order-statistic based "tramline" filter, which is robust against bright object distractors. Briefly, it is a generalized grey-level morphological top-hat filter, with two specialized structuring elements: first, a line oriented along the direction of the blood vessel; second, two "tram-lines" oriented in the same direction and lying to either side of the vessel. The filter response is the difference between the minimum intensity in the outer tramline structuring element and the maximum intensity level in the inner line structuring element. This filter has a strong response when the inner line is aligned within a blood vessel and the outer tramlines to either side of the vessel. The lines are each of length nine pixels, with the tramlines displaced five pixels to either side of the central line. The filter is rotated through twelve angles and the maximum response selected. In reality, maximum and minimum values are too noise-sensitive, and we use the third largest and third smallest values instead. We evaluated the algorithm on the Stare database [8], calculating the percentage of the gold-standard vasculature recovered by length, and the degree of false positive vessel detection as a percentage of gold-standard length (this contrasts with Hoover's evaluation which is per-pixel). Under these criteria, the Stare method achieves $57 \%$ sensitivity by length, with $8.5 \%$ false positive rate; the tram-line algorithm achieves $57.2 \%$ sensitivity and $2.9 \%$ false positive rate.

A typical vessel map is shown in figure 2. Once segmented, the vessel center lines are regularly sampled to provide points for diameter measurement. Diameter measurement algorithms, including ours, do not function correctly at major vessel branch points, crossing points, or where there are parallel vessels in close proximity or overlapping (where junction exponents are to be calculated the measurements are made just before and after the junction, not on it). Our algorithm inherently removes junction points and crossings, which is advantageous for some applications, such as venous beading detection, and disadvantageous for others (detection and measurement of junctions). The diameter measurement algorithm, however, is entirely separate from the segmentation algorithm. 


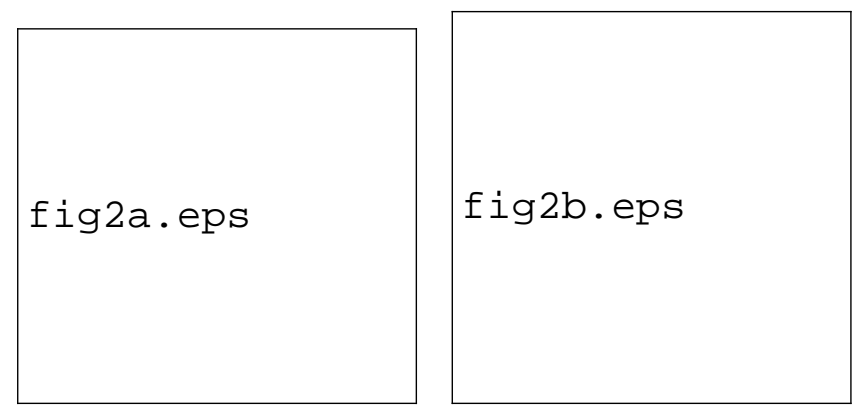

Fig. 2. Pre-processing a) Vessel map. b) Vessel map overlay.

\section{DiAMETER MEASUREMENT ALGORITHMS}

Vascular response to different physiological pathologies has been widely studied using a variety of methods. Measurements have been made both directly using ophthalmoscopy and indirectly using fundus photographs. Direct methods that require ophthalmoscopic observations are often inaccurate due to non-standardized illumination, low magnification, and a subjective bias [13]. More objective measurements are possible from fundus images in which the film transmission has been converted to intensity [14] [15] [16]. Converting the film transmission to intensity has been described as the only objective measure of the properties of the photographed eye; however, the observer has a restricted ability in discriminating between grey intensity levels [17], so that the measuring "by eye" directly from green channel intensity images is difficult and error prone.

As a result, a vascular cross-section is often presented in graphical form; see figure 3. The intensity level of the background retina is often different on either side of the vessel and the vessel often contains a bright reflex.

Due to pulse variations in vessel calibre, retinal photographs taken at the same fundus position will not yield the same vascular width, if exposed at different times. However, the use of relative widths (i.e. the ratios of individual widths to the average vessel width) can ensure reproducibility, as pulse characteristics, as with variable illumination conditions from incident light, are discounted in ratios. It is also important to note that, for automated diagnostic purposes, obtaining consistent measurements is more important than obtaining the actual vessel widths, as it is variations in widths along a vessel, or ratios of widths at difference points in the vessel, that 


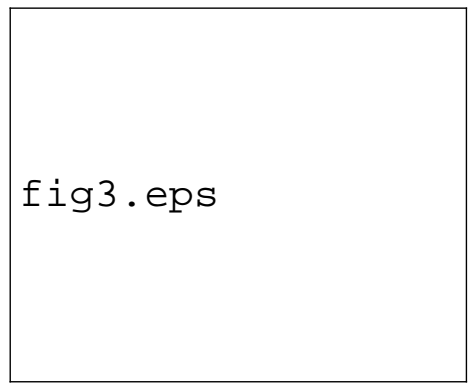

Fig. 3. Sample vessel profile.

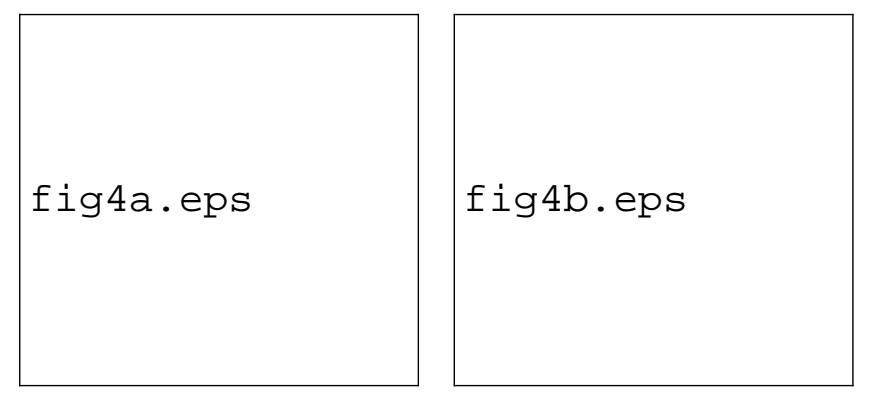

Fig. 4. a) Full width half maximum. b) Rectangular profile.

is of interest, rather than the absolute value of the width.

Several previous authors have presented algorithms for measuring vascular diameters. BrinchmannHansen et al [17] [18] measured widths of retinal vessels using micro densitometry and observed the importance of the central light reflex which prevents naïve approaches from working well. They presented an algorithm called Full Width Half Maximum (FWHM) to measure vascular diameters. This approach calculates a "half height point" on the left and right sides of the initial estimated mid-point of the profile. On each side, the minimum and maximum intensity levels are calculated, and the "half height point" is located where the profile crosses the mid point in intensity between the minimum and maximum. The FWHM estimate of the profile width is then the distance between these half height points; see figure 4a. This approach is also called Half Height Full Width (HHFW).

Gregson et al [19] introduced an alternative approach, comprising of a rectangular profile of a fixed height that is fitted to the profile data. The height is fixed to the difference between the minimum and maximum intensity values in the profile. The width of the rectangular profile is 
adjusted until the area under the rectangular profile is equal to the area under the profile data; see figure $4 b$.

Chapman et al [20], compares three methods of automated vascular measurements with manual recorded vessel diameters. A Gaussian function using non-linear regression, a Sobel edge detection algorithm and a sliding linear regression filter (SLRF) are all compared. Chapman states that using the Sobel edge detection algorithm to locate the blood vessel edges is unreliable due to a tendency to wrongly locate the central light reflex as the vessel edge. Chapman found the SLRF method to be most accurate and robust of these three methods; however, it is not practical for low-resolution images, as a minimum number of 10 pixels are required to estimate the slope by linear regression.

Chapman et al [20] and Zhou et al [21] have both experimented with Gaussian fitting functions to estimate vessel width. Chapman reported the Gaussian function fairing badly in comparison with the SLRF method. Zhou, on the other hand, reported promising results using a Gaussian model. Pedersen et al [3] presented an algorithm that used a cubic spline with 6 degrees of freedom to model the vascular profile and reduce noise. This method did not measure vessel diameters directly, but helped to reduce noise and relied upon Gregson's rectangular profile for width measurements. Gao et al [22] used a Difference-of-Gaussian to improve performance on vessels with a light reflex.

A number of authors have used matched filters to detect and segment blood vessels, including filters with Gaussian profiles. Gang et al [12] showed that the width control parameter of a gaussian profile matched-filter is linearly related to the actual blood vessel width.

The majority of the aforementioned approaches use a one-dimensional cross-sectional profile to measure the vascular diameter. Matched filters, in contrast, form a two-dimensional profile that is more resilient to poorly positioned profiles, and poorly defined vascular edges, but are applied at set sizes (typically in steps of one pixel). The proposed method uses a two-dimensional model with a Gaussian or Difference-of-Gaussian profile, which is iteratively optimized to best fit the observed vessel. This allows us to calculate the vessel width to sub-pixel accuracy, and the smoothing introduced by the two-dimensional nature of the profile improves this accuracy. In this paper the presented algorithm is compared to a Gaussian fitting function, HHFW and Gregson's rectangular profile and superior performance is demonstrated. 


\section{MethoD}

\section{A. Model definition}

The 2D model in its most basic form consists of an idealized straight vessel segment, with a Gaussian profile, $G_{1}$, orthogonal to the vessel direction, which can be optimized to closely map a vessel segment; see figure 5. The size and shape of the model is controlled by a small number of parameters, which can be adjusted to fit the model to the actual vessel profile; we use variable-metric optimization to find a good set of parameters. As it is important that the model mimics the vessel segment being measured as accurately as possible, a variation can be used to model the light reflex that is apparent in some vessel segments. This is achieved by subtracting a second small Gaussian curve, $G_{2}$, from the main one. The second Gaussian is oriented at the same angle as the first, and is independently adjustable allowing the overall model to fit well to both blood vessel column wall and light reflex. As not all vessel segments have a light reflex, both models with and without light reflex are fitted and the model with the best fit is selected.

The 2D model without light reflex (see figure 5) has a profile shape governed by equations 1 and 2.

$$
\begin{gathered}
\alpha=x \sin \theta-y \cos \theta-\mu \\
f_{x, y}=t-h_{1} e^{-s_{1} \alpha^{2}}
\end{gathered}
$$

where $x$ and $y$ map the profile data range in this example from -10 to +10 in increments of 1 . The model parameters are: $t$, the profile maximum; $h_{1}$, the height of the Gaussian; $s_{1}$, the width

of the Gaussian, equal to $\frac{1}{\sigma_{1}^{2}} ; \theta$, the orientation of the model; and $\mu$, the offset of the Gaussian center from the initial center estimate, orthogonal to the orientation. Figure 5 illustrates the model and parameters (barring $\mu$ ).

The two-dimensional model with light reflex shares the same base parameters as its non light reflex counterpart. The light reflex variation to the core archetype consists of a Gaussian curve $G_{2}$ subtracted from $G_{1}$. Additional parameters are included to control the shape and size of the light reflex. These parameters, $h_{2}$ and $s_{2}$, set the height and width of the Gaussian $G_{2}$ respectively. The model with light reflex is expressed by equation 3, and illustrated in figure 6 . 


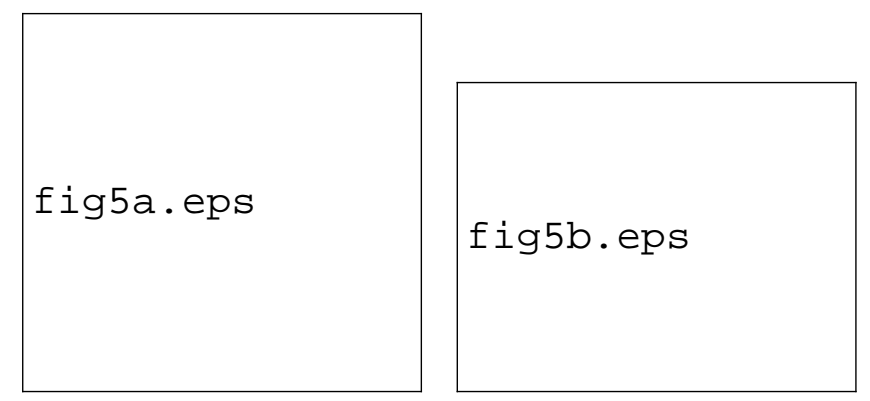

Fig. 5. Model without Light Reflex. a) Cross-section of Profile. b) Two-dimensional Representation.

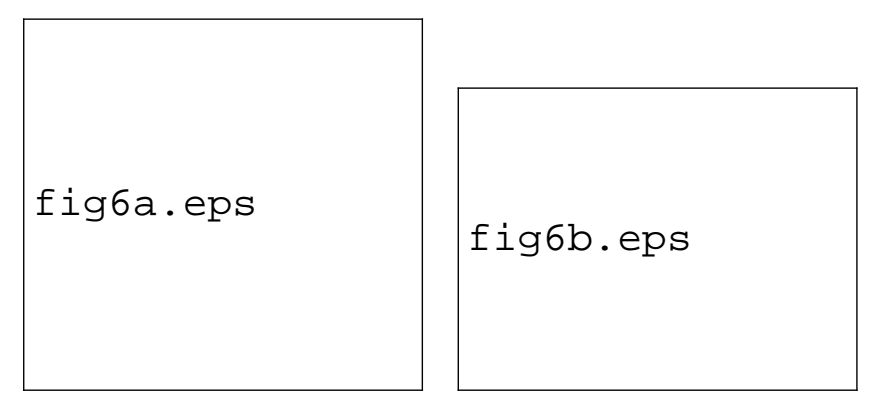

Fig. 6. Model with Light Reflex. a) One-dimensional Representation. b) Two-dimensional Representation.

$$
f_{x, y}=t-\left(h_{1} e^{-s_{1} \alpha 2}-h_{2} e^{-s_{2} \alpha 2}\right)
$$

\section{B. Optimization}

\section{B.1 Two stage optimization process}

A two-stage optimization process is used to orientate and shape the models to fit a $2 \mathrm{D}$ vessel segment, within an oriented rectangular region of interest centered at an initial point, $\left(p_{x}, p_{y}\right)$, taken from the vascular profile, and oriented along the vessel line as estimated from previous and next points in the vascular profile; see figure 7. In the first stage, a relatively long vascular region of interest is formed, the model width is fixed to a fairly broad value, and the algorithm determines the exact orientation, offset and height. In the second stage, a shorter region of interest is formed, the orientation is fixed, and the width, offset and height are determined to 
greater accuracy. Models both with and without a light reflex are optimized at each stage, the model which best fits the vessel segment is selected, and the width is calculated from the final parameters.

The two stage process is necessary for two reasons. First, the short model is poor at determining the angle as it lacks sufficient support along the vessel line. Second, it is prone to "default errors," where the model completely fails to capture the vessel shape (e.g. by forming a very wide, flat Gaussian fitted on one side to the background region) if the initial settings are too far from the correct values. The first stage, however, is very robust. It handles well the entire range of typical vessel widths, and inaccuracies in the initial vessel center line up to $66 \%$ (at a $95 \%$ confidence level there is no statistically significant change in vessel width measurements up to this disarticulation). In our experience it is almost unknown for the algorithms that determine the initial line to exceed a $66 \%$ error in center line determination. Once the first stage has determined the angle, and reasonable starting values for the other parameters, the second stage determines a more accurate local width measurement, and is very reliable.

\section{B.2 Vascular region of interest and mask}

The 2D model is fitted to a local section of vessel, within a rectangular region of interest (ROI) that is oriented at the initial estimated angle, $\theta$, of the blood vessel. The use of an oriented ROI is important as the background intensity varies fairly quickly, and there may be other vessel segments nearby; in early work using a square region of interest oriented with the image axes, we found that such features in the corners of the ROI could have detrimental effects. For computational convenience we calculate the bounding rectangle of the ROI, and form a mask to identify the ROI; see figure 7 . We then perform the calculations over the bounding rectangle, and multiply through by the mask, $z_{x, y}$, to ensure that only the pixels within the ROI take effect. Given a ROI of length $L$ (along the vessel orientation) and width $W$ (orthogonal to it), the mask is applied to a region area from $p_{x}-X$ to $p_{x}+X$ and $p_{y}-Y$ to $p_{y}+Y$, where $X=(L \cos \theta+W \sin \theta), Y=(L \sin \theta+W \cos \theta)$.

The size of the ROI depends upon the stage of model optimization. In the first stage a $15 \times 15$ ROI is used to enable the 2D model to fix the local angle against a sufficiently long vessel segment and to find the vessel "line" despite noise. In the second phase a $5 \times 20$ ROI allows the 2D model obtain a good localized diameter measurement. With the average width of larger vessels 


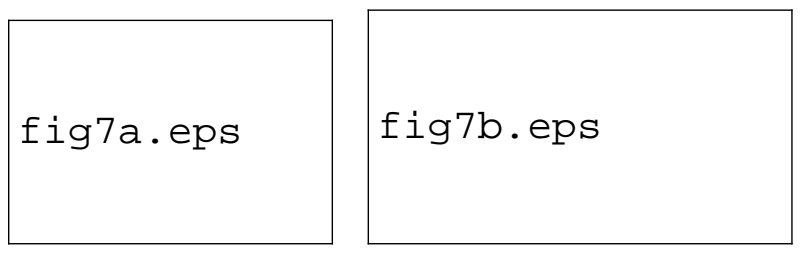

Fig. 7. Vascular model. a) Vessel segments points. b) Mask and Region of Interest

in a $760 \times 570$ fundus images being $6-9$ pixels, both ROI are large enough to allow flexibility for off centered and beaded vessels, and to accommodate sufficient areas of the background.

\section{B.3 Initial settings of model}

The initial settings of the model parameters are extremely important. Bad initial settings may result in poor parameter optimization and an undesirable fit between model and vessel, leading to an inaccurate width measurement.

The initial settings for all of the parameters have been determined by trial and error. We set $h_{1}=1.5 d, h_{2}=0.5 d$ where $d$ is the difference between the minimum and maximum intensity values within the region of interest. The offset $\mu$ is set to zero. The width of the Gaussian $G_{1}, s_{1}$, is set to 0.1 , giving a medium width with respect to the expected range, allowing the model to fit to both larger and smaller profiles. The width of the Gaussian $G_{2}, s_{2}$, is set to 0.8. The value of $\theta$ is determined by the vector connecting neighboring vessel segment points $P_{n-1}$ to $P_{n+1}$ (this gives a good enough starting value for the model to be optimized to the true angle).

\section{B.4 Parameter Optimization}

To effectively optimize the model, we used Quasi-Newton (variable metric) minimization [23], a fast iterative algorithm that adjusts the model from the initial settings, by minimizing an error function that summarizes the goodness of fit of the current model. The procedure also requires a function giving the gradient of the error function with respect to each of the model parameters. The error function we use is the sum-squared disparity between the model estimate and actual intensity values at the centers of the pixels in the region of interest, as expressed by equation 4.

$$
E=\frac{1}{2} \sum_{x=-X}^{X} \sum_{y=-Y}^{Y} z_{x, y}\left(f_{x, y}-I_{p_{x}+x, p_{y}+y}\right)^{2}
$$


Where $I$ is the image, $f$ the model and $z$ is the region of interest mask. The model is substituted by equations 5 and 6 for two-dimensional models without and with light reflex respectively.

The formulae for the gradients of this error function with respect to the model parameters are given in Appendix A.

\section{Determining the model width}

Once the model has been fitted, its parameters are used to estimate the vessel width. As previously noted, for further automated analysis it is important that the width measurement be consistent, rather than that it coincide with the user-perceived edge of the blood column, and any consistent bias can easily be removed.

For the single Gaussian model, the sigma parameter is the most appropriate estimator; this coincides with the inflection point on the slope of the vessel side. For the Difference-of-Gaussian model, we also use the inflection point on the side slope; however, there is no simple analytical expression that yields its position. Instead, we use a simple line search procedure to locate a zero of the second derivative of the model, and the offset of this inflexion point defines the vessel width.

\section{TESTING AND RESUlts}

\section{A. Benchmarking Method}

The refractive index and the distance between the retina and the camera lens affect vascular diameters observed from a retinal camera [20]. Absolute measures of diameter are therefore difficult to obtain. The only appropriate method of testing and comparing a new measurement technique is to compare measurements from the same vessel. To this end we tested the accuracy and precision of the presented algorithm using 100 gold standard widths obtained from five high-resolution fundus images with a 45 degree field of view, and photographed using a Canon 60uv fundus camera. All 100 vessel widths were selected from non-tortuous vessel segments between bifurcations. The dimensions of the high-resolution images were $3300 \times 2600$ pixels, approximately four times larger than the standard $760 \times 570$ lower resolution fundus counterparts used in our screening programs. By manually measuring widths on the high-resolution images, and down-sampling the images to test the algorithms at low resolution, we obtain gold standard widths with sub-pixel accuracy. It is against this benchmark that the accuracy and precision of 


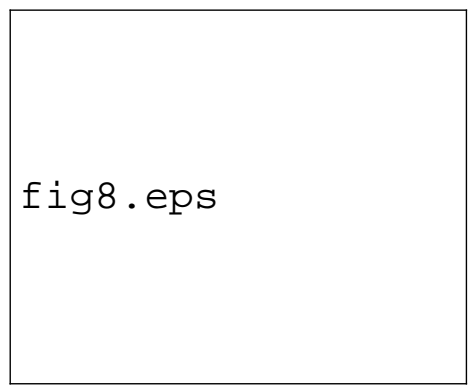

Fig. 8. Kick Points

the algorithm was measured.

The width measurements from the aforementioned high-resolution images were taken at segment points and fixed angles derived by the algorithm during the blood vessel segmentation and profile data extraction phases. At segment point $P$ the angle $\theta$ was used to plot an orthogonal profile line to the vessel centreline. The profile length from the full size fundus images was set to 80 pixels, 4 times larger than that used in the scaled image; the profile was constructed using bilinear interpolation.

A sophisticated technique called "kick points", due to Rassam et al [1], was used to manually calculate the vascular widths from the full size images. The "kick points" are visible points on the slopes of intensity profiles, defined as the first skew points. Kick points occur where the wall meets the most lateral extent of the blood column; the horizontal distance between the kick points indicates the blood column width (see figure 8) .

Rassam et al [1], states that the kick points approach is more accurate than finding the width at half the height; however, kick points are not always visible. If the blood column and the vessel wall have the same optical densities then kick points will not appear. To observe the kick points, the photograph must be high resolution and well focused, as the shape of the intensity curve changes with focusing. This makes the detection of the kick points difficult but has no effect on the degree of separation between points. On our images, kick points were visible on the high-resolution images, but not on the down-sampled versions.

The sections of the images used, vessel locations, measurement points and gold standard width are available from http://facs.lincoln.ac.uk/Research/LowellDiameter.htm, to allow other authors to compare with our method. 


\section{B. Models tested}

A comparison was made between the presented algorithm and three other well-established approaches. The algorithms of Brinchmann-Hansen and Heier's full width half maximum [18], Gregson et al's rectangular profile [19] and Zhou et al's Gaussian model [21] were implemented using exactly the same intensity profiles calculated at the same segment points and angles as the 2D model. As previously discussed, the 2D algorithm is given a rectangular region of interest centered at the initial profile center point. In contrast, the three benchmark algorithms use a onedimensional profile which we determine by bi-linear interpolation along the profile direction.

One justification for using a $2 \mathrm{D}$ model is that this smooths the local diameter estimates. As an additional comparison, we also collected the average width measurement from an ensemble of $1 \mathrm{D}$ profiles spanning the same range as the $2 \mathrm{D}$ model, to see if similar results can be obtained just by averaging $1 \mathrm{D}$ profiles. The reasoning behind this study was to confirm that the $2 \mathrm{D}$ model was not just behaving like a series of 1D Gaussian models. In this test, if any of the ensemble 1D profile parameters were outside a specified range (indicating a failure in model fitting) that profile was excluded from the averaging process (one-dimensional profiles are more susceptible to such instabilities than the 2D model).

\section{Results}

In this application it is more important that the algorithm produces results that are precise (low error variance) than accurate (low mean error), as consistency is more significant than absolute diameter. Any consistent bias is easily subtracted to determine the true width. However if the results fluctuate, no compensation is possible. For this reason, the error standard deviation was used rather than the mean to assess the performance of the algorithms. The 100 width estimates, $\omega_{i}$, returned from each algorithm were subtracted from 100 appropriately scaled user benchmark widths, $\psi_{i}$, giving a width difference $\phi_{i}=\omega_{i}-\psi_{i}$. The standard deviation of the width differences was then taken, $\sigma_{\phi}$.

We verified that the difference in standard deviations between the $2 \mathrm{D}$ model and the alternative approaches is statistically significant. An F-test was performed to test the null hypothesis that the variance of the $2 \mathrm{D}$ model is equal to that of the next best model, the "average 1D" model. The F-test uses the variance ratio $\left(0.5^{2} / 0.34^{2}=2.212\right)$ to test for significant differences in variances. 
TABLE I

STANDARD DEVIATION OF WIDTH DIFFERENCE AT A SCALING OF 4

\begin{tabular}{|c|c|}
\hline & $\sigma_{\phi}$ \\
\hline 2D Model & 0.34 \\
Avg 1D Gaussian & 0.5 \\
1D Gaussian & 0.58 \\
HHFM & 0.62 \\
Gregson & 0.84 \\
\hline
\end{tabular}

With 99 degrees of freedom in each sample, the F-test critical values are 1.39 at $95 \%$ confidence, 1.6 at $99 \%$ confidence. Consequently we reject the null hypothesis, and conclude that the 2D method is better than the average 1D model, even at the $99 \%$ confidence level. Similar pairwise comparisons indicate the superiority of the 2D model over the other alternative algorithms.

Zhou et al [21] claimed that their approach, the 1D Gaussian model, was more precise than other algorithms, including FWHM. Our study supports this claim. However, the presented 2D algorithm is $32 \%$ more precise again. The 1D Gaussian model is also prone to failure to converge sufficiently enough to obtain a sensible width reading ( $4 \%$ of diameters in our tests). Using an ensemble of 1D models eradicates this problem, and so the ensemble estimate out-performed the single Gaussian model.

It is worth noting that the $2 \mathrm{D}$ model algorithm is accurate, on average, to 0.34 of a pixel; given that the manual process underlying the ground-truth measurements for the tests is accurate to only 0.25 pixels this is excellent performance. If a more accurate ground-truth were available it is possible that even better performance could be demonstrated.(see figure 9a).

\section{Effectiveness of the light reflex model}

To assess the effectiveness of the model's light reflex in producing a more precise measurement, 20 benchmark widths that include a visible light reflex were used to compare against widths taken from the model with and without light reflex. It was found that the $2 \mathrm{D}$ model with light reflex gave results that were $13 \%$ more precise than given by its basic model counterpart in cases where the vessel segment included a light reflex. Although the improvement in the 
performance by using a light reflex model is marginal, it is statistically significant.

\section{E. Effect of high curvature on precision}

To evaluate the effect of high curvature vessel segments on the model's precision, 28 widths from high curvature points were calculated. It was found that at the $95 \%$ confidence level, there was no statistically significant change in width precision between normal and high curvature widths (see figure 9b). This gives us some confidence that the algorithm will work to a reasonable extent even on somewhat tortuous vessels, although we note that both segmentation and measurement are liable to fail on profoundly looped vessels, where the vessel crosses itself or two segments run very closely in parallel. Detailed measurement of neovascular vessels remains an open question.

\section{F. Effect of beaded vessels on precision}

To assess the consequence of dramatic width changes within a vascular segment, 40 widths from beaded vessels were taken. The algorithm deteriorated the standard deviation increasing by $\sigma_{\phi}=0.124$ pixels to $\sigma_{\phi}=0.464$ pixels (a statistically significant difference at $95 \%$ confidence). The other algorithms suffered a similar deterioration in precision with Gregson's method performing second best at $\sigma_{\phi}=0.771$ pixels. Comparing these two models show that the 2D model is better than Gregson's method and the findings were statistically significant at $99 \%$ confidence. (see figure 9c).

\section{CONCLUSION AND DISCUSSION}

We have presented an algorithm to automatically extract width measurements from retinal vessels. This plays a critical part in the structured analysis of the retina, and is potentially useful in the automated diagnosis of eye diseases such as diabetic retinopathy and arteriosclerosis.

Comparing the vascular widths returned by the presented algorithm, HHFW, Gregson and a 1D Gaussian, it was shown that the presented model is more precise by over $30 \%$. This is a considerable amount when small changes in the vascular diameter can alter the outcome of searches for pathology.

During model fitting, a sophisticated optimization (Quasi-Newton) strategy was used, and appropriate energy function derivatives introduced. 

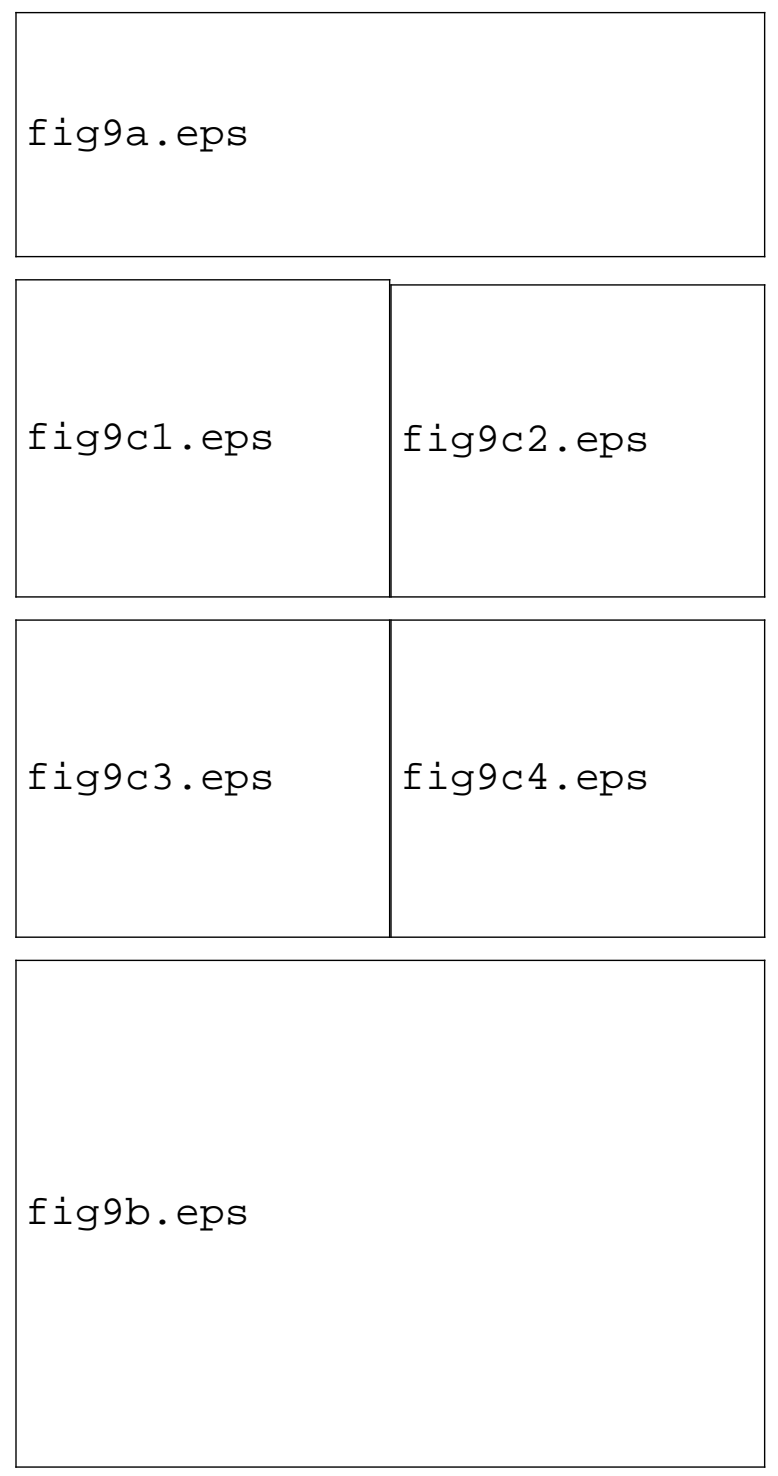

Fig. 9. Detected widths on: Top) straight; Middle) curved; Bottom) beaded vessel segment.

The use of a 2D model circumvents problems caused by noisy data such as small vessel branches and introduces a degree of smoothing that helps to improve the model fit. We have shown that the inclusion of a Difference-of-Gaussians model improves performance over a single Gaussian where there is a visible light reflex. Finally, we note that the algorithm is robust enough to make sub-pixel accurate measurements on relatively low-resolution images, where vessels are only a few pixels wide. 


\section{APPENDIX A}

The Quasi-Newton (BFGS) optimization procedure uses a gradient descent approach, and consequently requires the calculation of the gradient of the error function with respect to the model parameters.

The differential of the error function (see equation 4) with respect to the model $f$ is given by:

$$
\frac{\delta E}{\delta f}=\sum_{x=-X}^{X} \sum_{y=-Y}^{Y} z_{x, y}\left(f_{x, y}-I_{p_{x}+x, p_{y}+y}\right)
$$

Individual terms of the error gradient are derived by using the chain rule, multiplying equation 5 by the differentials of the model with respect to individual parameters.

The partial differentials for the parameters $h_{1}, h_{2}, s_{1}, s_{2}, \mu$ and $\theta$ (see equations 1 and 3 ) are expressed below:

$$
\begin{gathered}
\frac{\delta f}{\delta h_{1}}=-e^{-s_{1} \alpha^{2}} \\
\frac{\delta f}{\delta h_{2}}=e^{-s_{2} \alpha^{2}} \\
\frac{\delta f}{\delta s_{1}}=h_{1} \alpha^{2} e^{-s_{1} \alpha^{2}} \\
\frac{\delta f}{\delta s_{2}}=-h_{2} \alpha^{2} e^{-s_{2} \alpha^{2}} \\
\frac{\delta f}{\delta \theta}=2 \alpha(-x \sin \theta+y \cos \theta)\left(h_{1} s_{1} e^{-s_{1} \alpha^{2}}-h_{2} s_{2} e^{-s_{2} \alpha^{2}}\right)
\end{gathered}
$$

(10)

$$
\frac{\delta f}{\delta \mu}=2 \alpha\left(-h_{1} s_{1} e^{-s_{1} \alpha^{2}}+h_{2} s_{2} e^{-s_{2} \alpha^{2}}\right)
$$




\section{ACKNOWLEDGMENT}

\section{This project was supported by Diabetes UK, Project Grant No. BDA:RD00/0002033.}

The authors would like to thank the referees for their detailed and useful suggestions, which have helped to greatly improve this paper.

\section{REFERENCES}

[1] S.M.B. Rassam, V. Patel, 0. Brinchmann-Hansen, O. Engvold, and E.M. Kohner, "Accurate vessel width measurement from fundus photographs: a new concept,” British Journal of Ophthalmology, vol. 78, pp. 24-29, 1994.

[2] Early treatment diabetic retinopathy study research group, "Fundus photographic risk factors for progression of diabetic retinopathy," Ophthalmology, vol. 98, pp. 823-833, 1991.

[3] L. Pedersen, M. Grunkin, B. Ersboll, K. Madsen, et al., "Quantitative measurement of changes in retinal vessel diameter in ocular fundus images," Pattern Recognition Letters, vol. 21, pp. 1215-1223, 2000.

[4] A.R. Sharrett, L.D. Hubbard, L.S. Cooper, P.D. Sorlie, et al., "Retinal arteriolar diameters and elevated blood pressure: the atherosclerosis risk in communities study," American Journal Epidemiology, vol. 150, pp. 263-270, 1999.

[5] T.Y. Wong, R. Klein, B.E. Klein, J.M. Tielsch, et al., "Retinal microvascular abnormalities and their relationship with hypertension, cardiovascular disease and mortality," Survey of Ophthalmology, vol. 46, no. 59-80, 2001.

[6] N. Chapman, G. Dell'omo, M.S. Sartini, and N. Witt, "Peripheral vascular disease is associated with abnormal arteriolar diameter relationships at bifurcations in the human retina," Clinical Science (London), vol. 103, pp. 111-116, 2002.

[7] T.Y. Wong, R. Klein, D.J. Couper, L.S. Cooper, et al., "Retinal microvascular abnormalities and incident stroke: the atherosclerosis risk in communities study," Lancet, vol. 358, pp. 1134-1140, 2001.

[8] A. Hoover, V. Kouznetsova, and M. Goldbaum, "Locating the blood vessels in retinal images by piecewise threshold probing of a matched filter response," IEEE Transactions on Medical Imaging, vol. 19, pp. 203-210, 2000.

[9] S. Chaudhuri, S. Chatterjee, N. Katz, M. Nelson, and M. Goldbaum, "Detection of blood vessels in retinal images using two-dimensional matched filters," IEEE Transactions on Medical Imaging, vol. 8, pp. 263-369, 1989.

[10] O. Brinchmann-Hansen and H. Heier, “The apparent and true width of the blood column," Acta Ophthalmologica, Supplement, vol. 179, pp. 29-32, 1986.

[11] B.D. Thackray and A.C. Nelson, "Semi-automatic segmentation of vascular network images using a rotating structuring element (rose) with mathematical morphology and dual feature thresholding," Pattern Recognition, vol. 15, pp. 431-443, 1982.

[12] L. Gang, O. Chutatape, and S.M. Krishnan, "Detection and measurement of retinal vessels in fundus images using amplitude modified second-order gaussian filter," IEEE Transactions on Biomedical Engineering, vol. 49, no. 2, February 2002.

[13] H. Neame, "A method of estimating the calibre of retinal arteries in the living eye by means of opthalmoscope, illustrated results in some normal and pathological cases," Trans. Ophthalmological Societies of the United Kingdom, vol. 56, pp. $155-162,1936$.

[14] T. Behrendt, "Scanning densitometer for photographic fundus measurements," American Journal of Ophthalmology, vol. 62, pp. 689-693, 1966.

[15] A. Kagan, E. Aurell, and G. Tibblin, "Signs in the fundus oculi and arterial hypertension. unconventional assessment and significance," Bull WHO, vol. 36, pp. 231-241, 1967.

[16] J.V. Hodge, J.C. Parr, and G.F.S. Spears, "Comparison of methods of measuring vessel widths on retinal photographs and 
the effect of fluorescein injection on apparent retinal vessel calibre," American Journal of Ophthalmology, vol. 68, pp. 1060-1068, 1969.

[17] O. Brinchmann-Hansen and O. Engvold, "Microphotometry of the blood column and light streak on retinal vessels in fundus photographs," Acta Ophthalmologica, Supplement, vol. 179, pp. 9-19, 1986.

[18] O. Brinchmann-Hansen and H. Heier, "Theoretical relationships between light streak characteristics and optical properties of retinal vessels," Acta Ophthalmologica, Supplement, vol. 179, pp. 33-37, 1986.

[19] P.H. Gregson, Z. Shen, R.C. Scott, and V. Kozousek, “Automated grading of venous beading," Computers and Biomedical Research, vol. 28, pp. 291-304, 2000.

[20] N. Chapman, N. Witt, X. Goa, A. Bharath, A.V. Stanton, S.A. Thom, and A.D. Hughes, "Computer algorithms for the automated measurements of retinal arteriolar diameters," British Journal of Ophthalmology, vol. 85, pp. 75-79, 2001.

[21] L. Zhou, M.S. Rzeszotarski, L.J. Singerman, and J.M. Chokreff", "The detection and quantification of retinopathy using digital angiograms," IEEE Transactions on Medical Imaging, vol. 13, no. 4, December 1994.

[22] X.W. Goa, A. Bharath, A. Stanton, A. Hughes, N. Chapman, and S. Thom, "Quantification and characterisation of artieries in retinal images," Computer Methods and Programs in Biomedecine, vol. 63, pp. 133-146, 2000.

[23] C.M. Bishop, Neural Networks for Pattern Recognition, Clarendon Press, Oxford, 1995. 


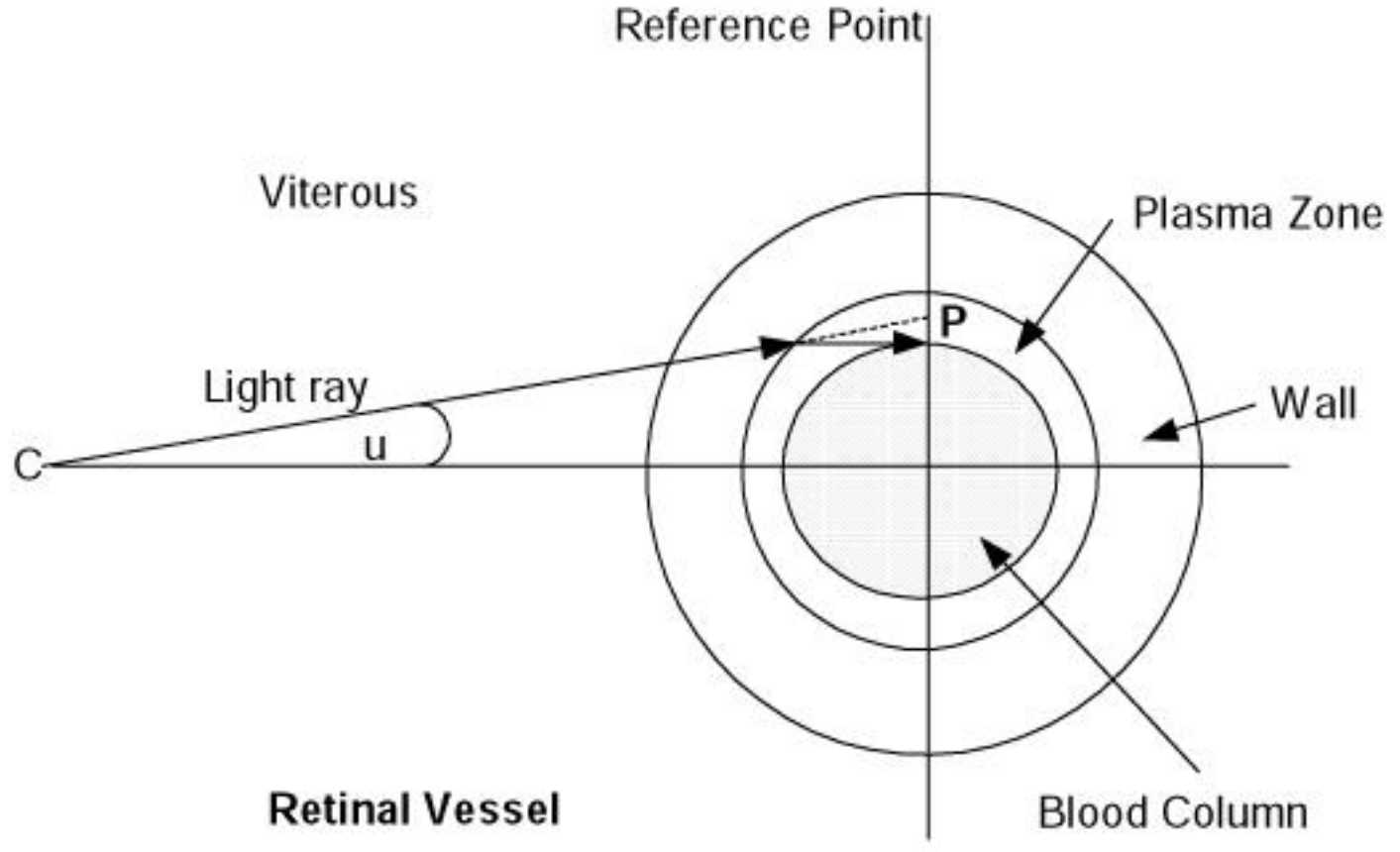

fig1.eps 
24

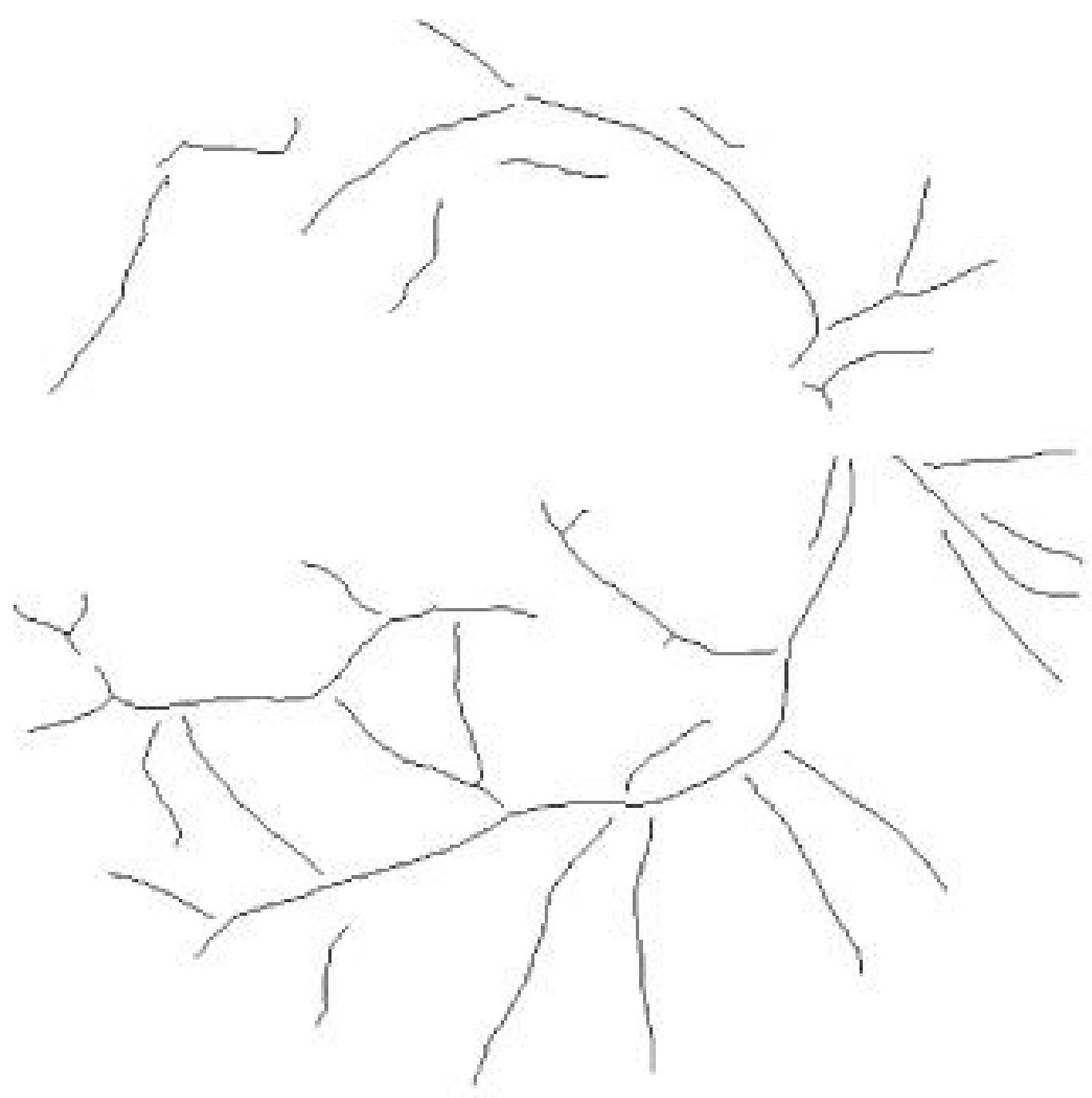

fig2a.eps 


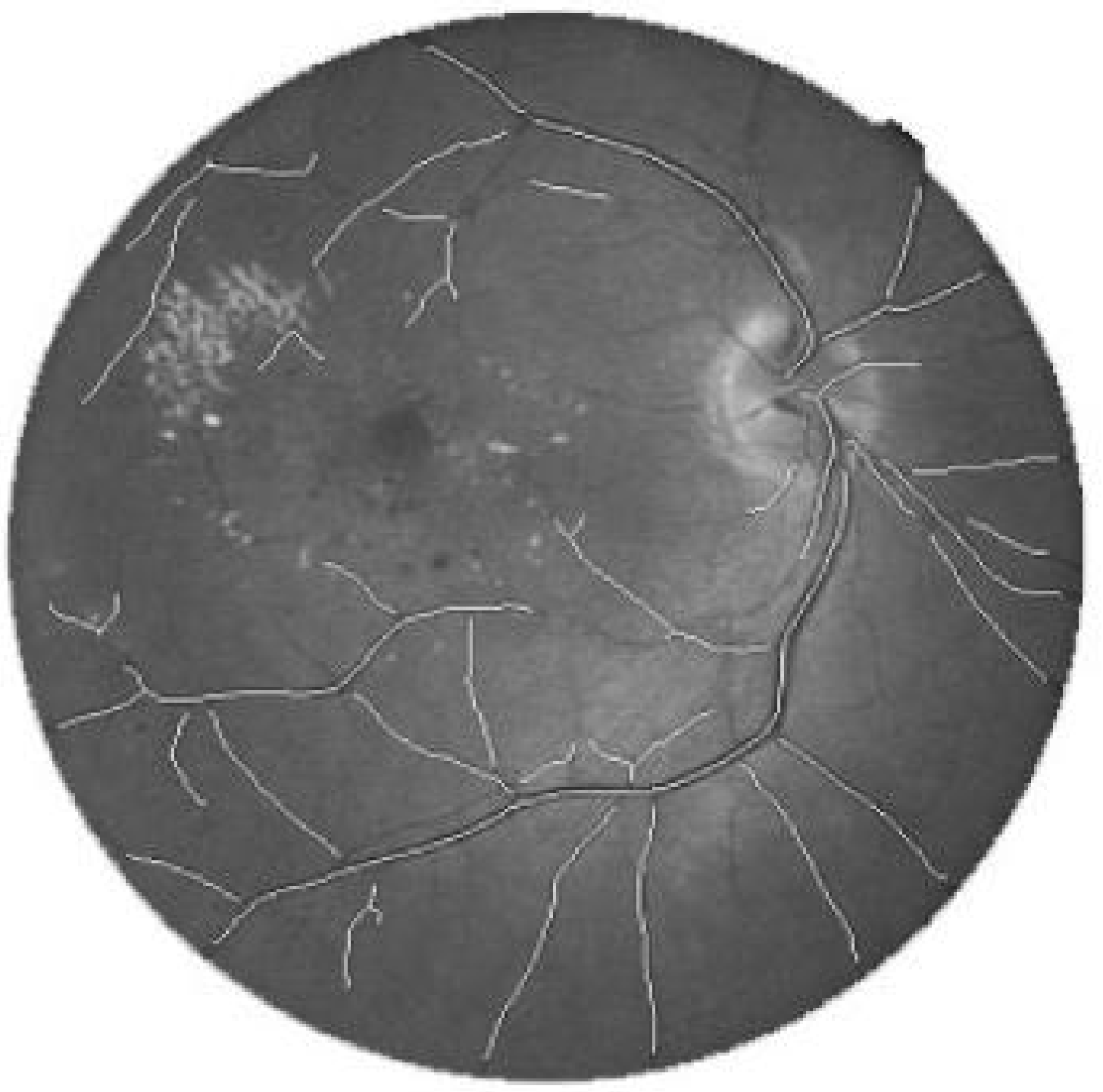

fig2b.eps 


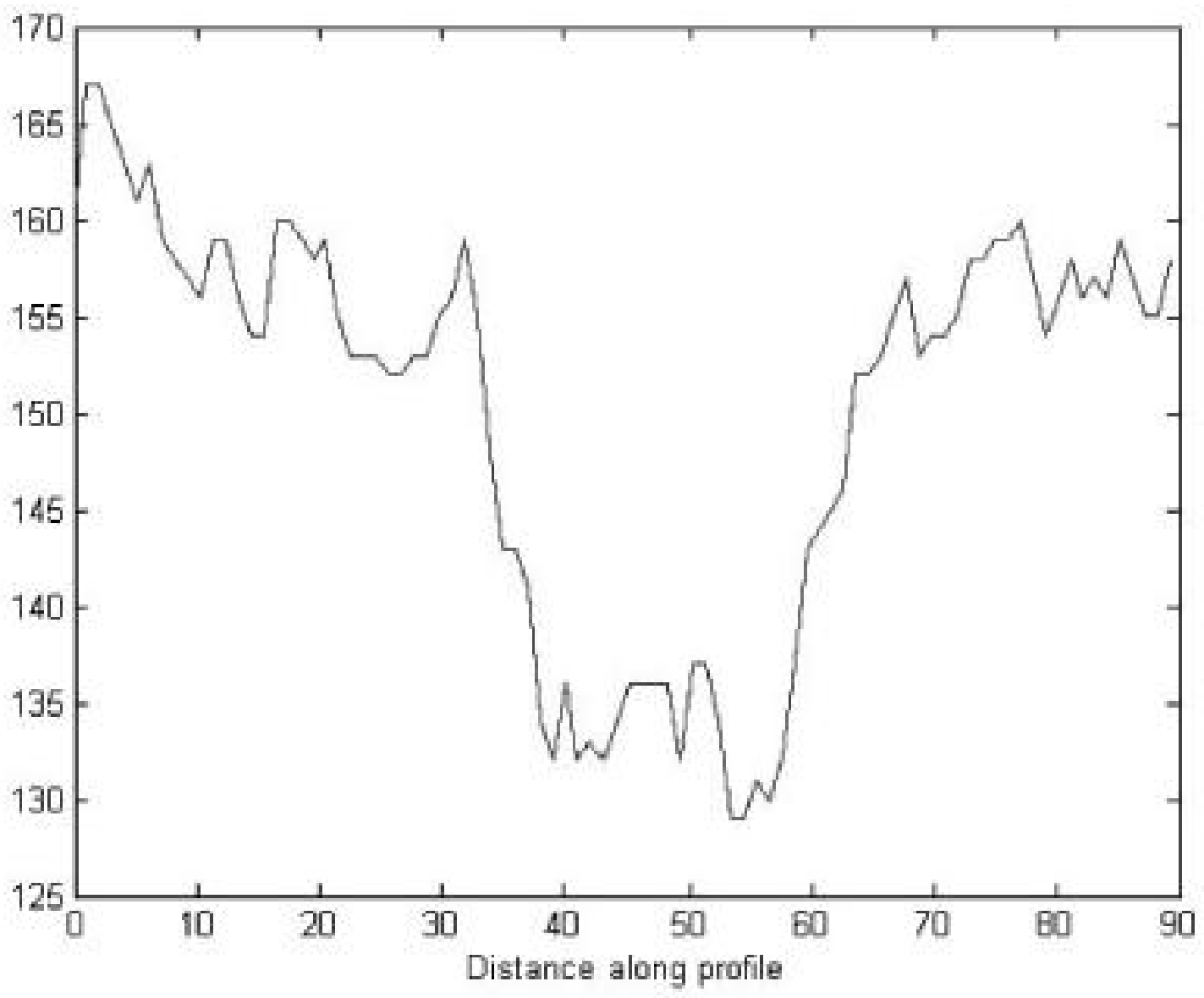

fig3.eps 


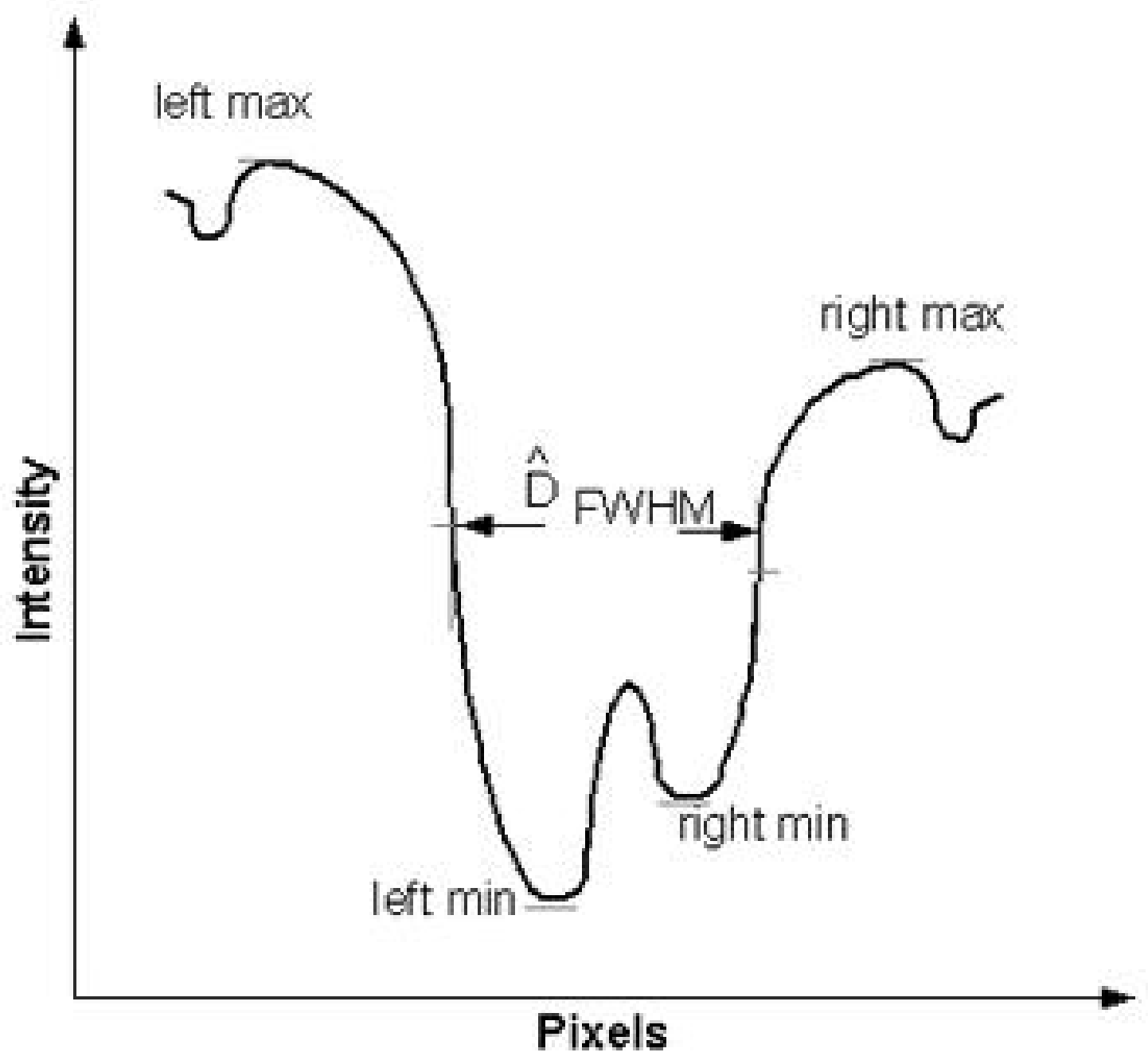

fig4a.eps 


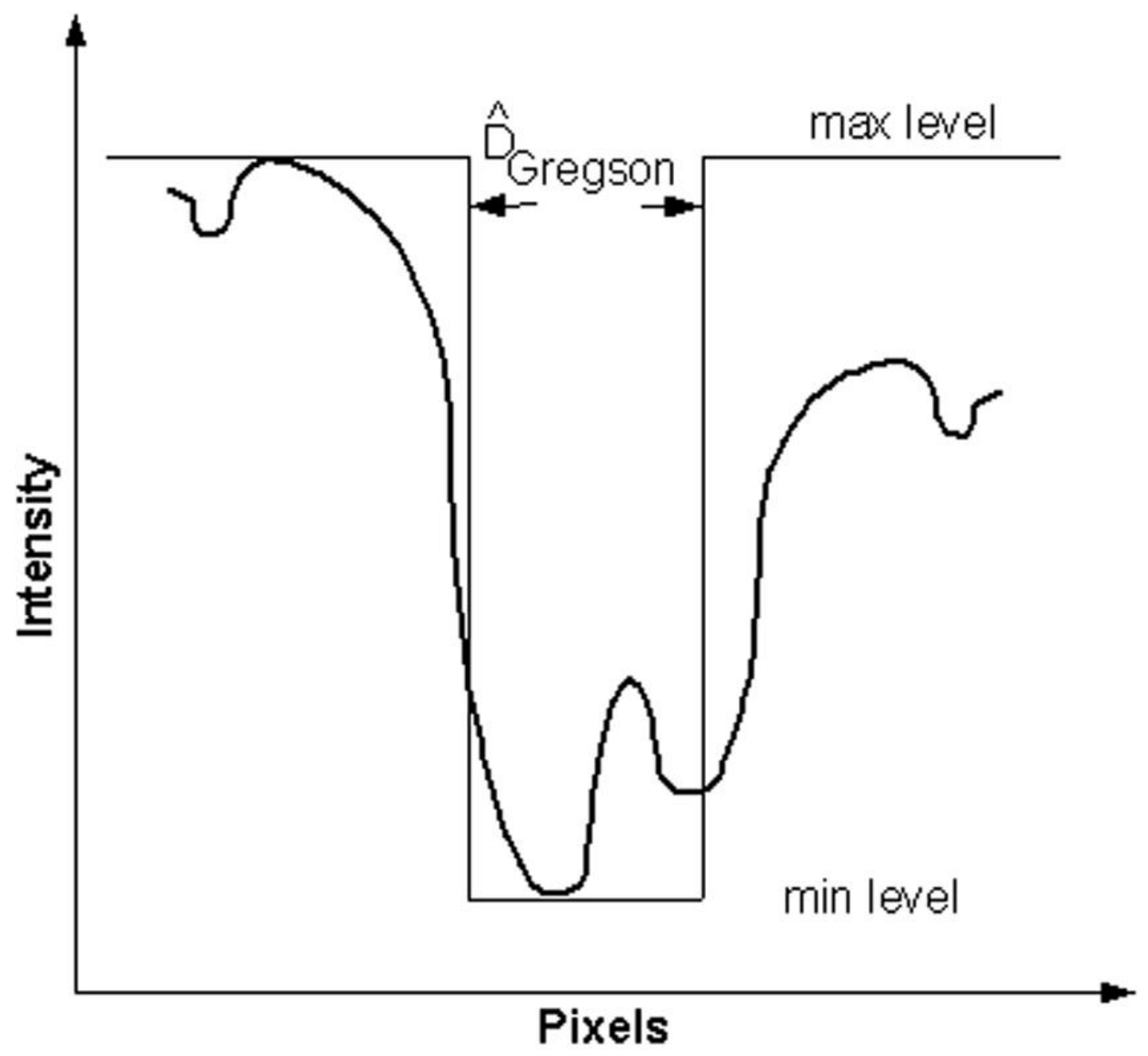

fig4b.eps 


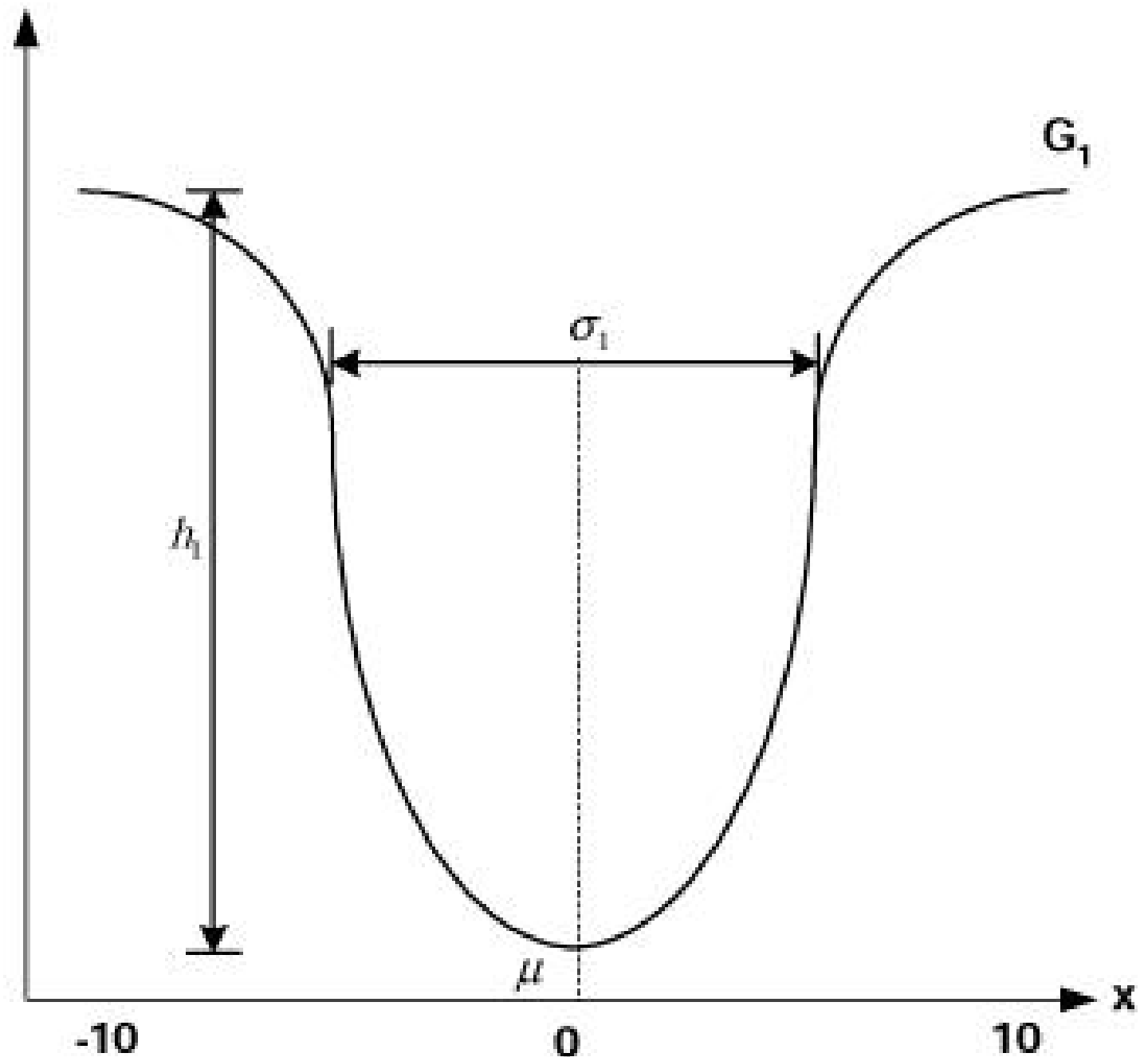

fig5a.eps 


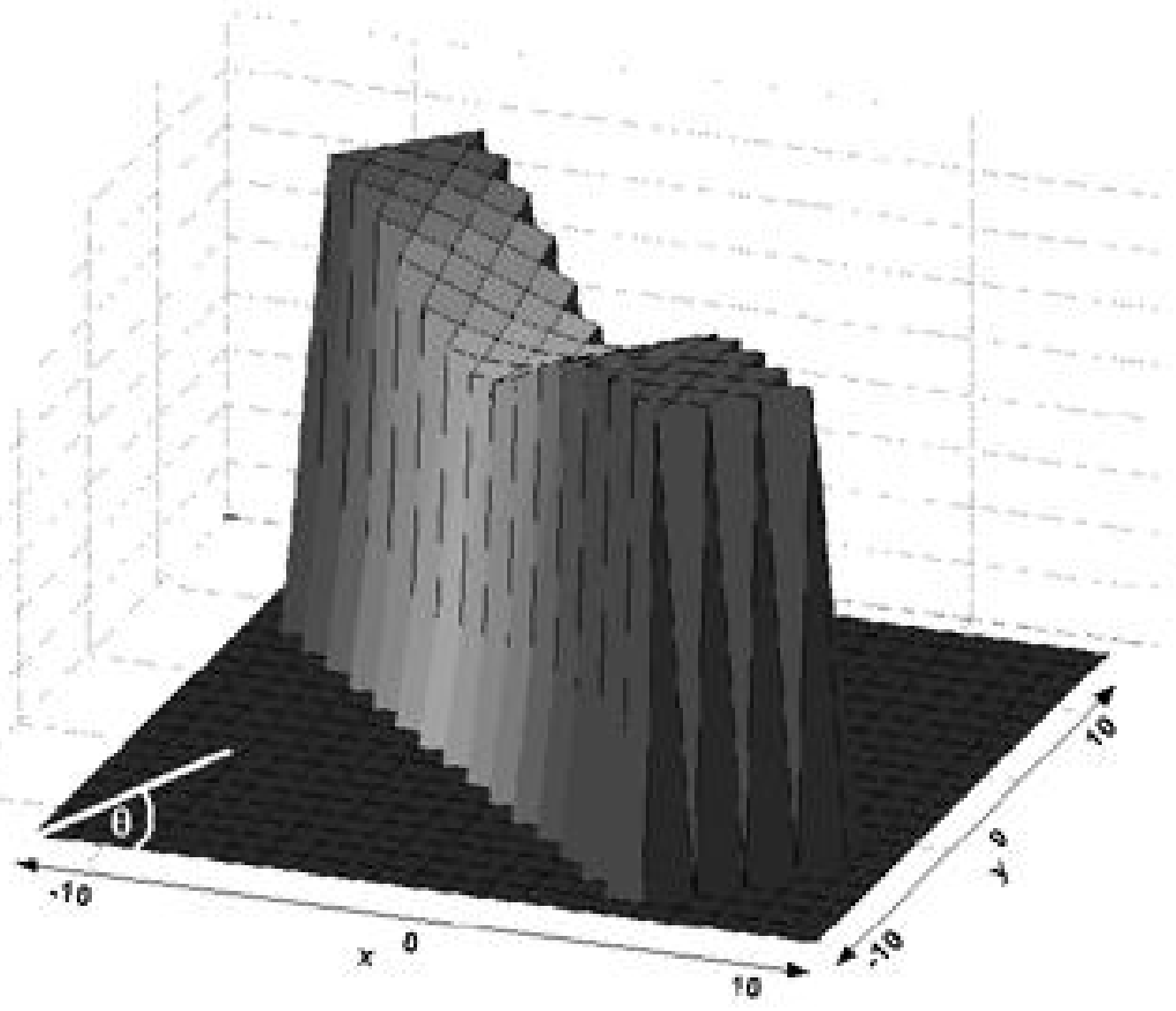

fig5b.eps 


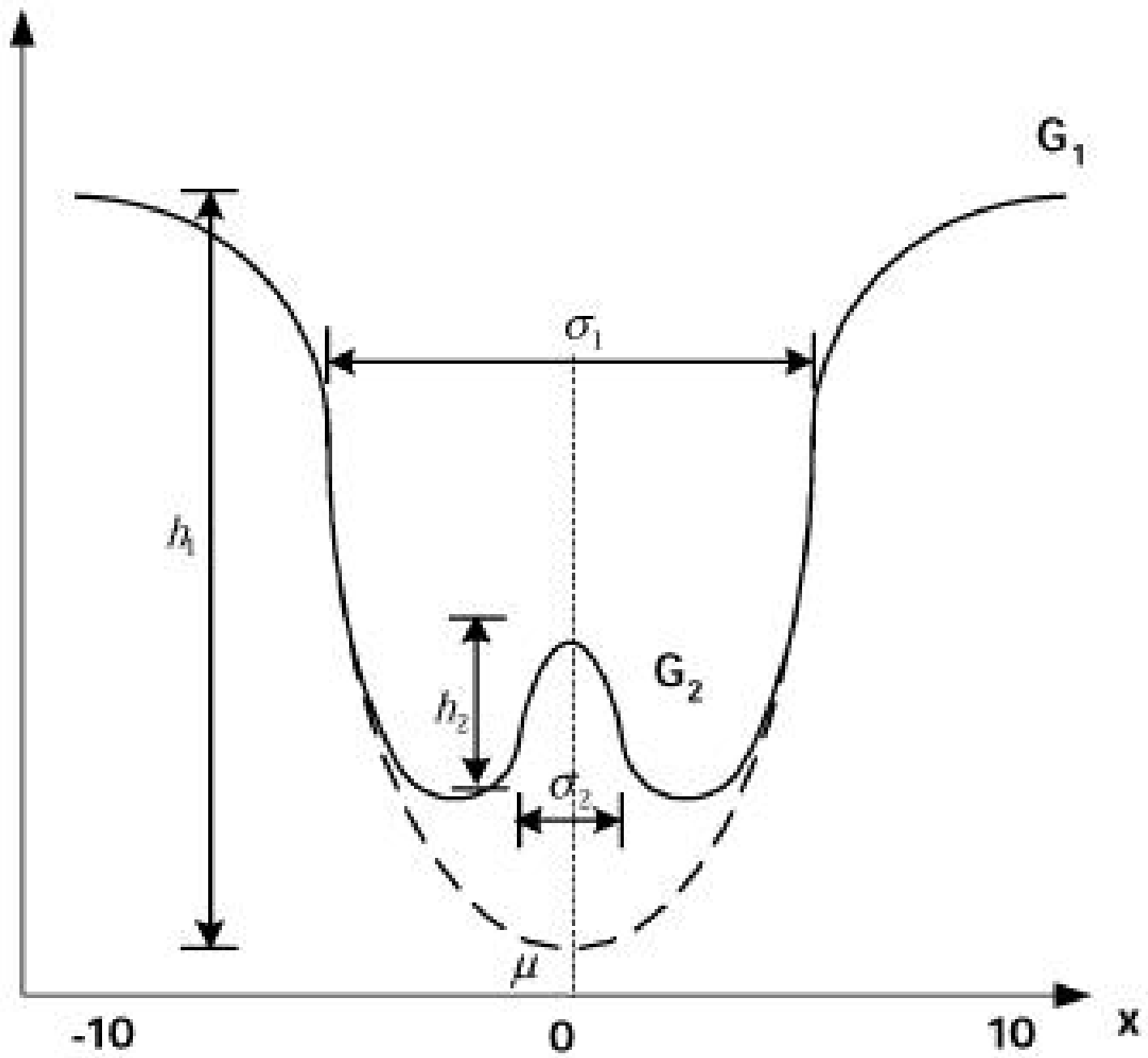

fig6a.eps 


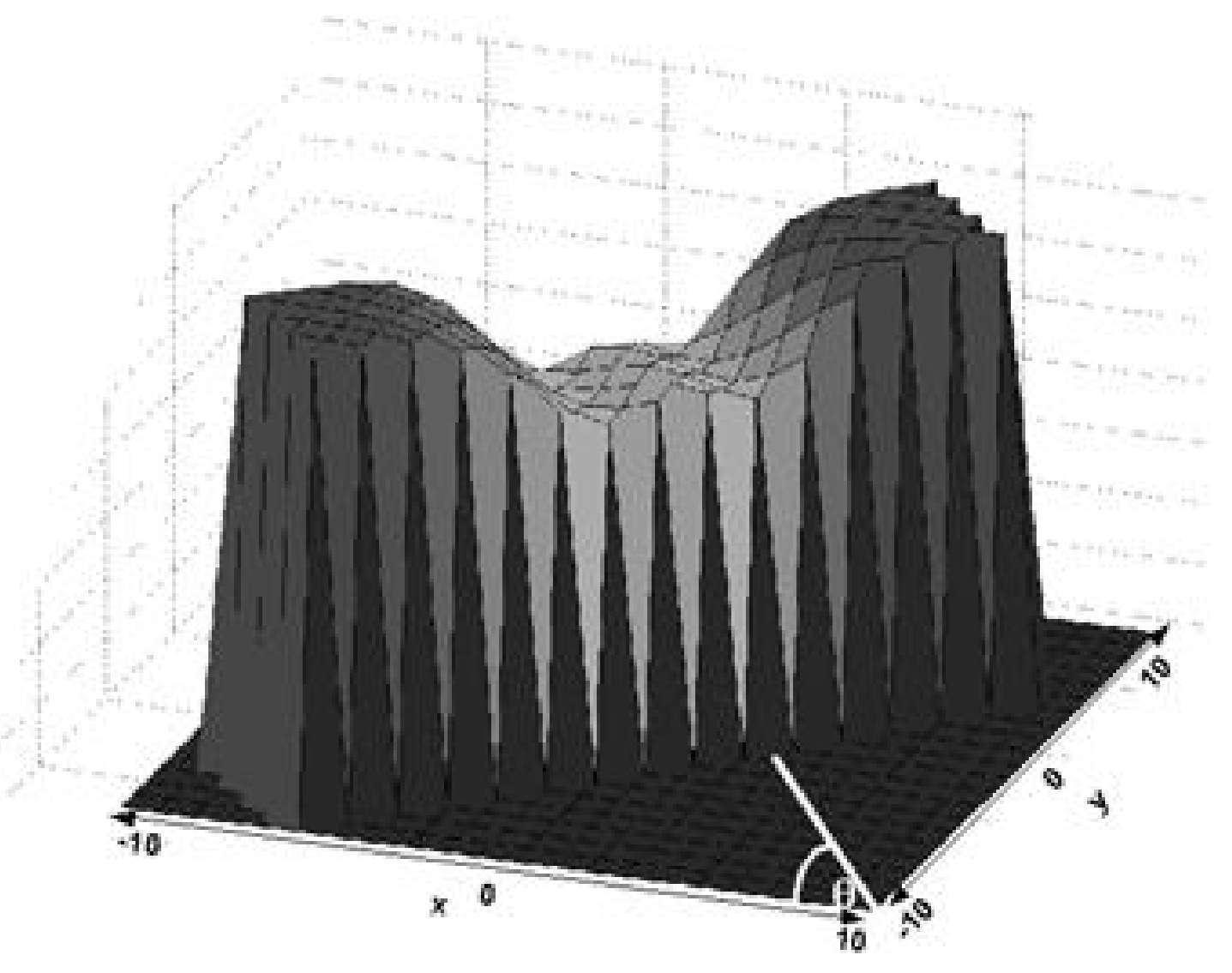

fig6b.eps 


\section{Centreline}

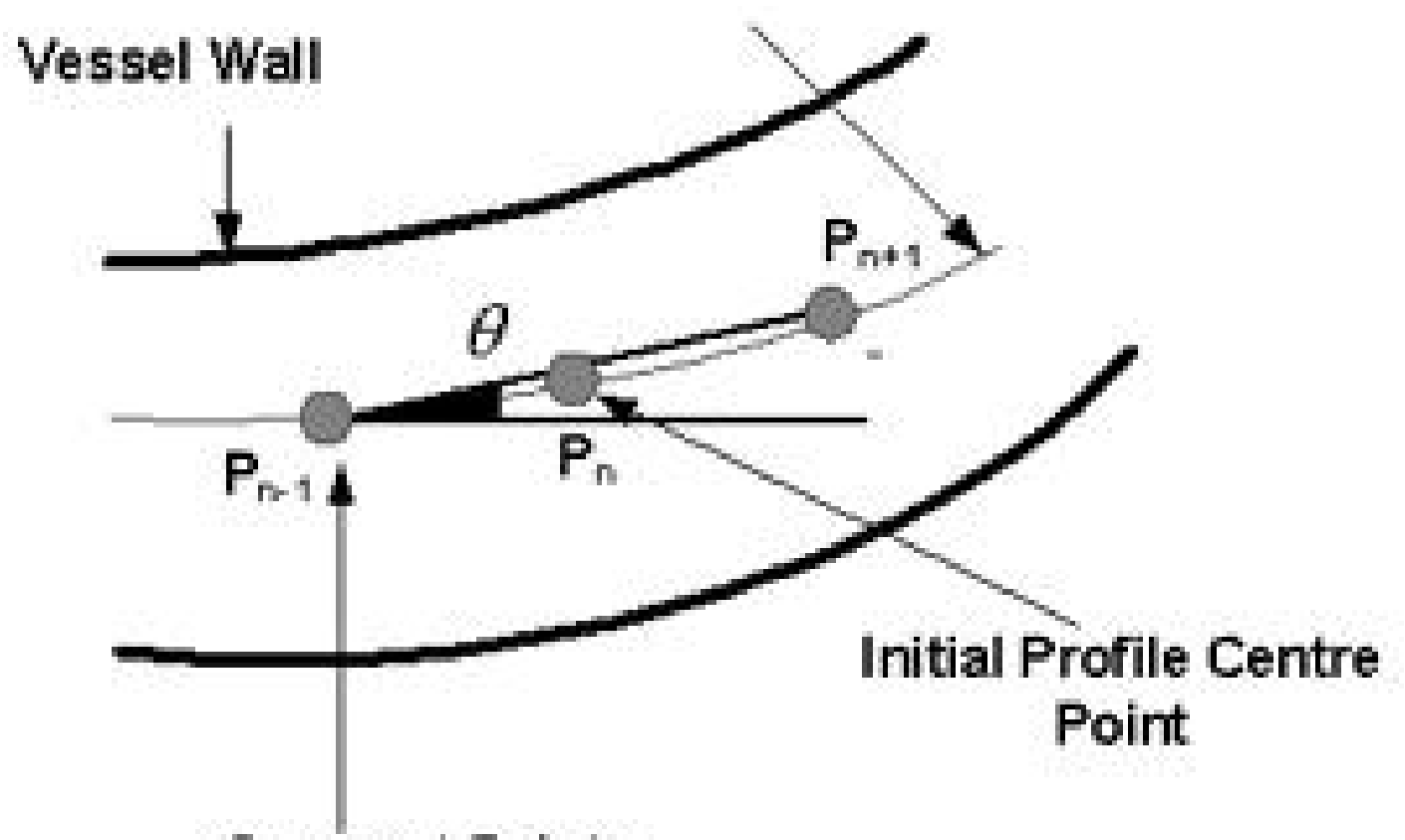

Segment Points

fig7a.eps 


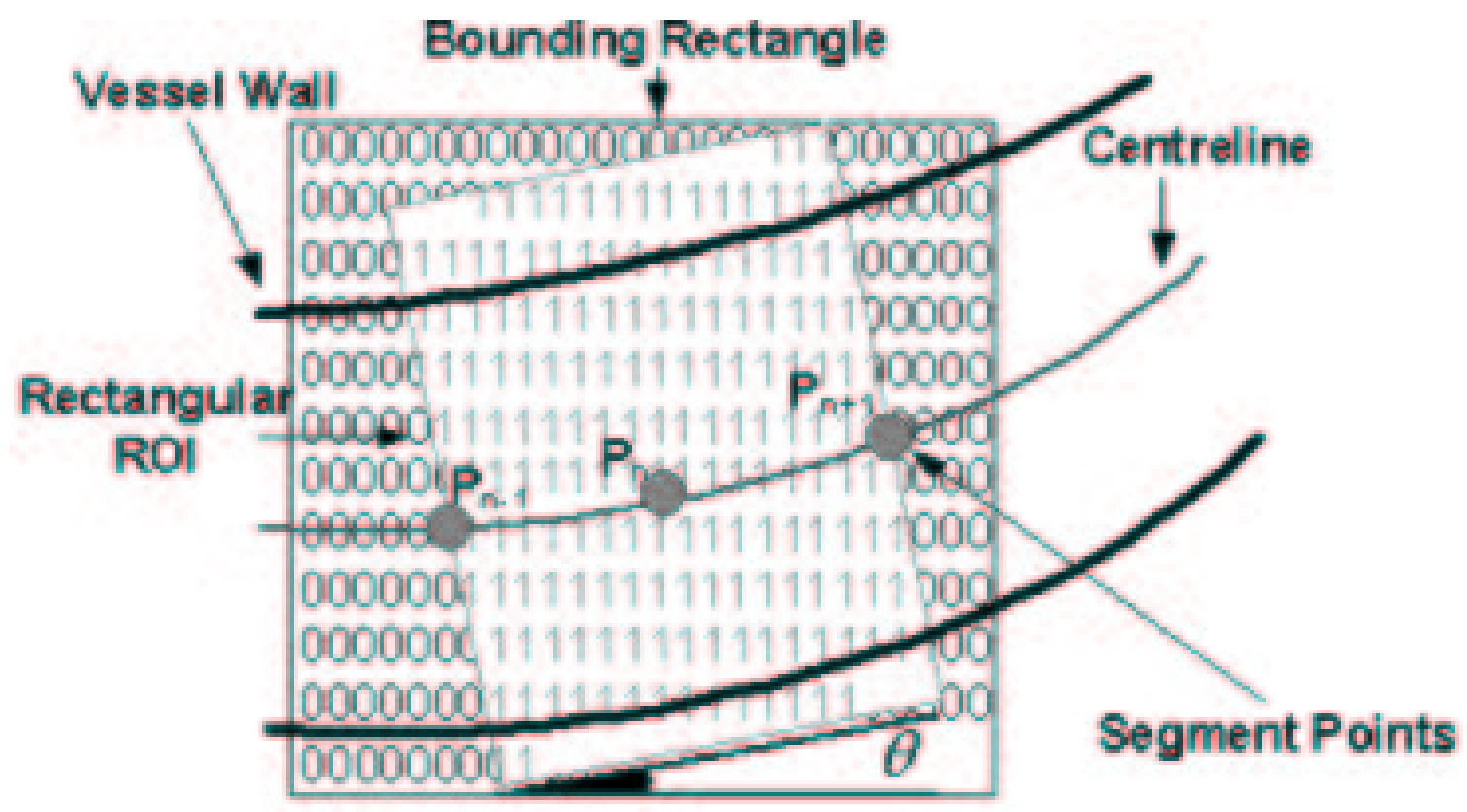

fig7b.eps 


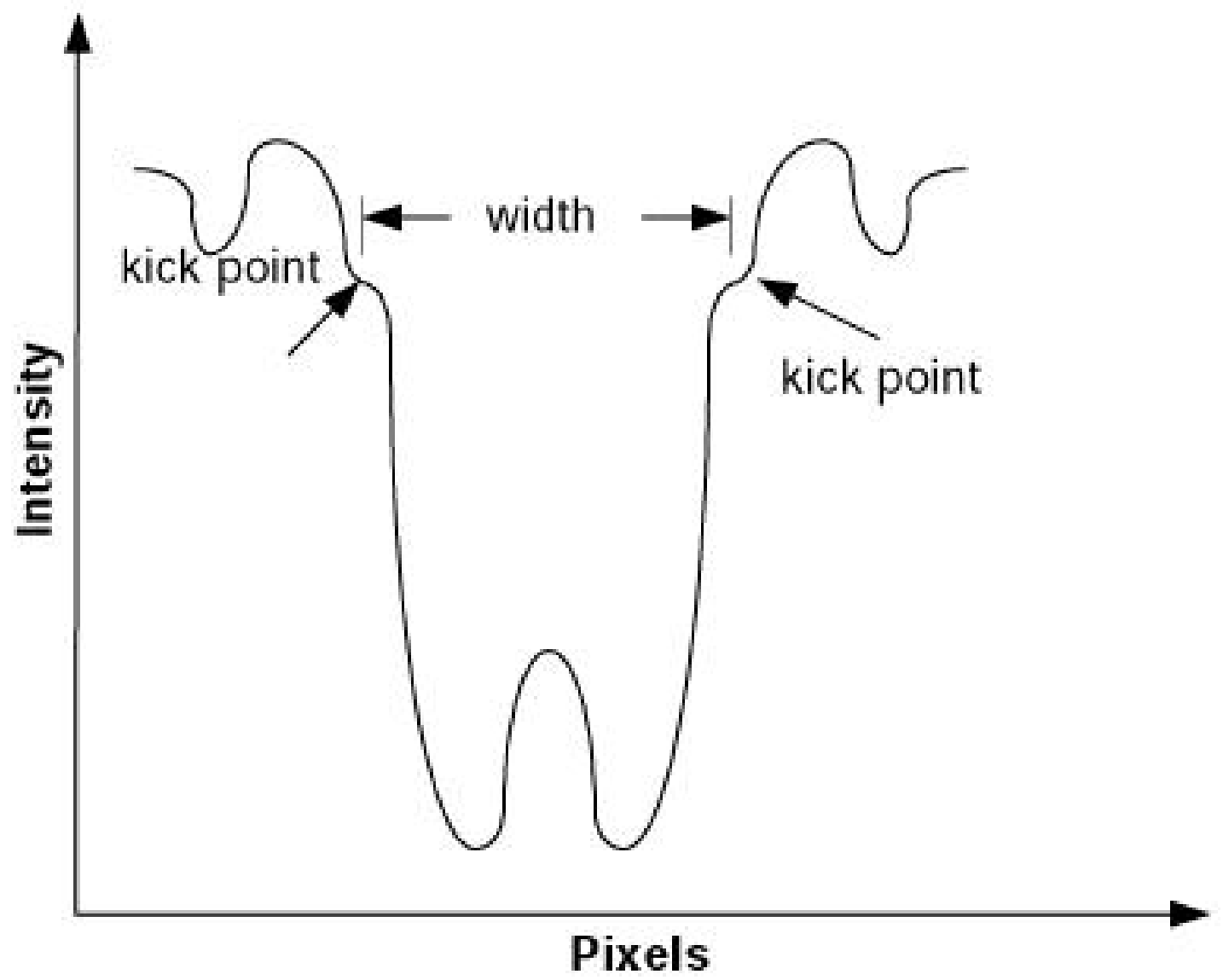

fig8.eps 


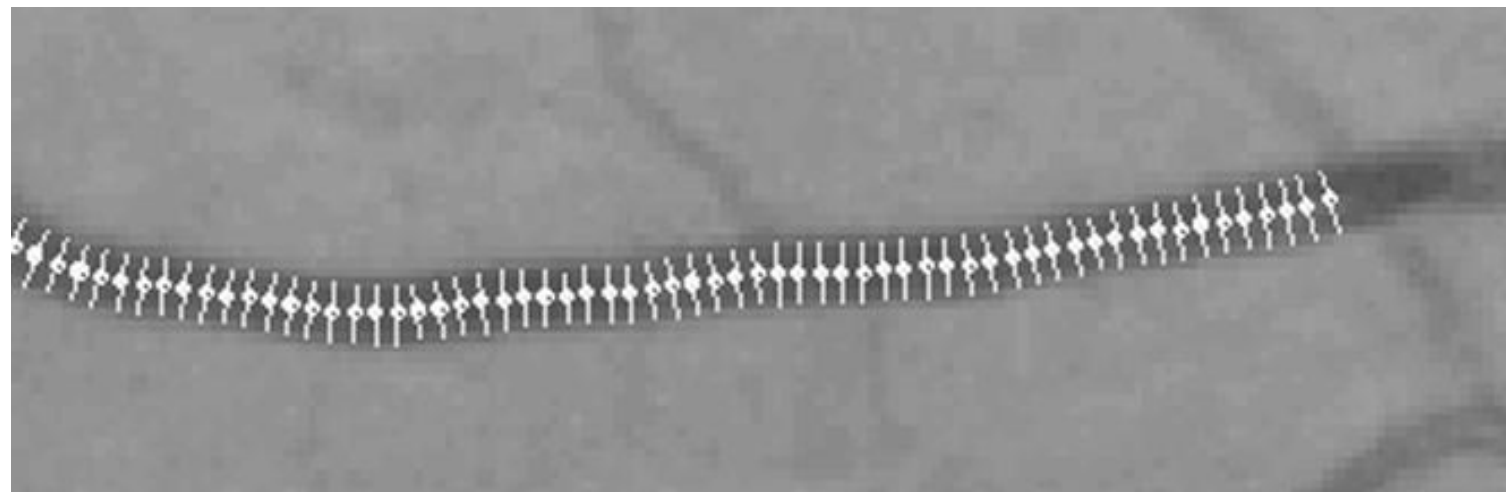

fig9a.eps 


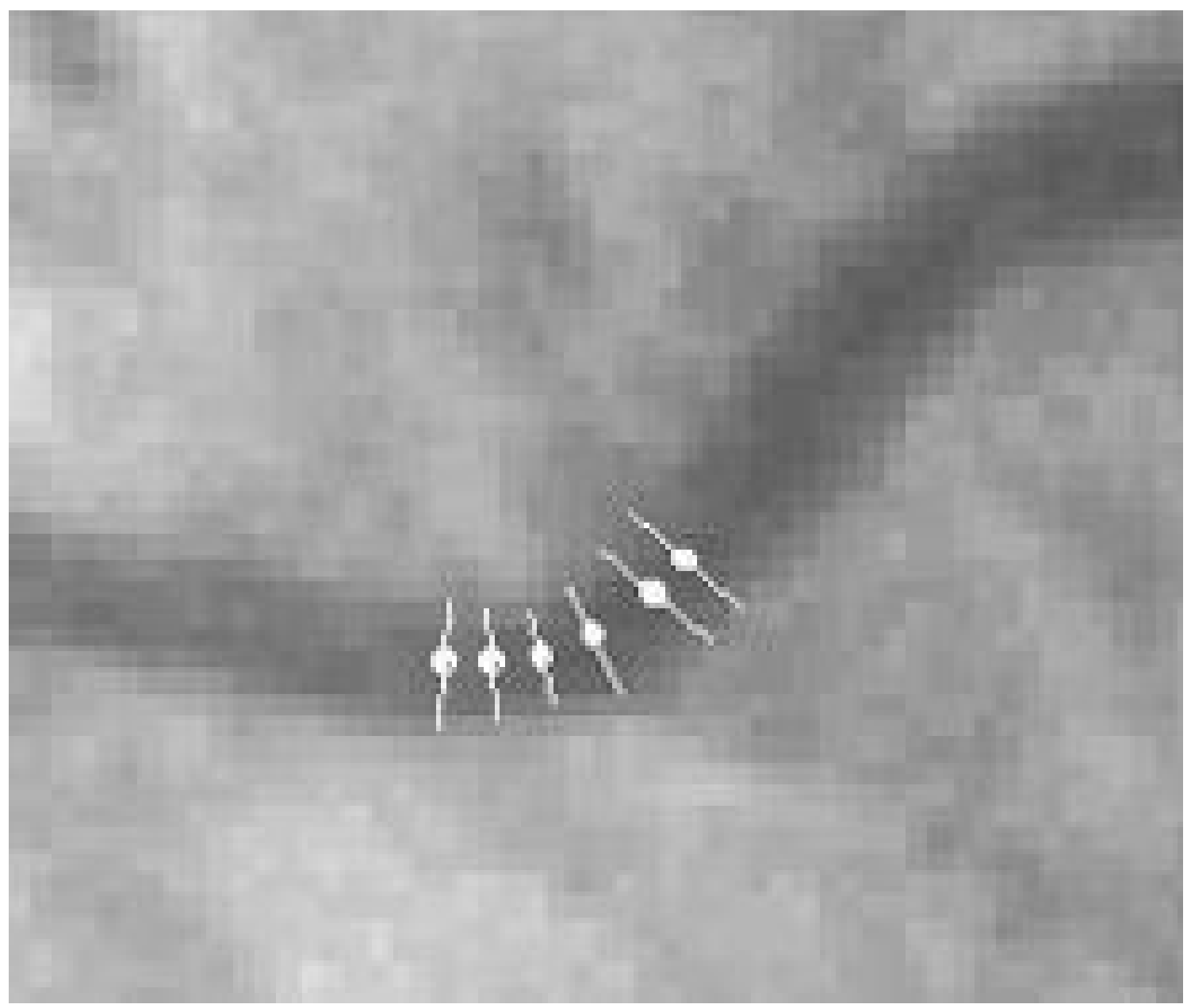

fig9c1.eps 


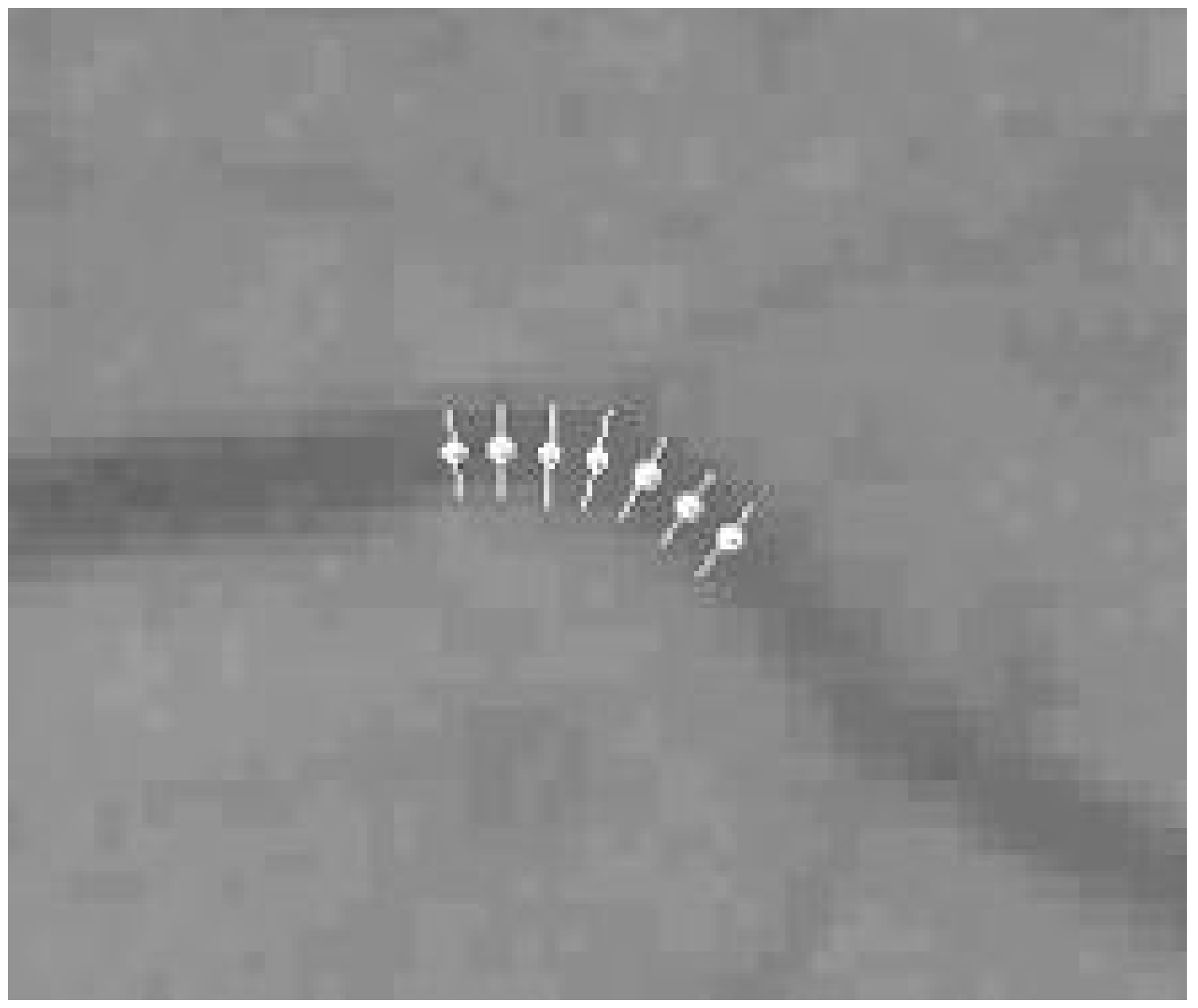

fig9c2.eps 


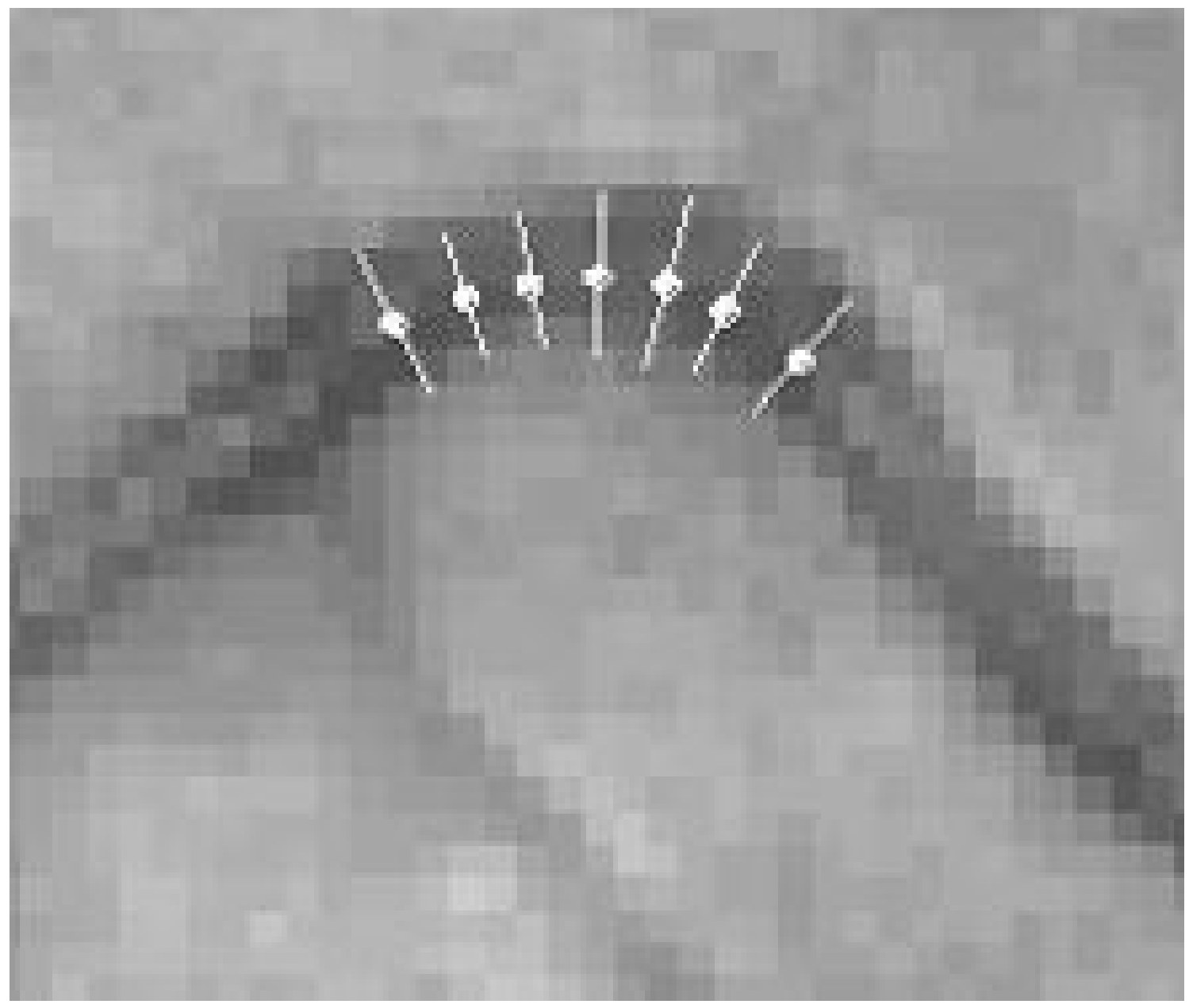

fig9c3.eps 


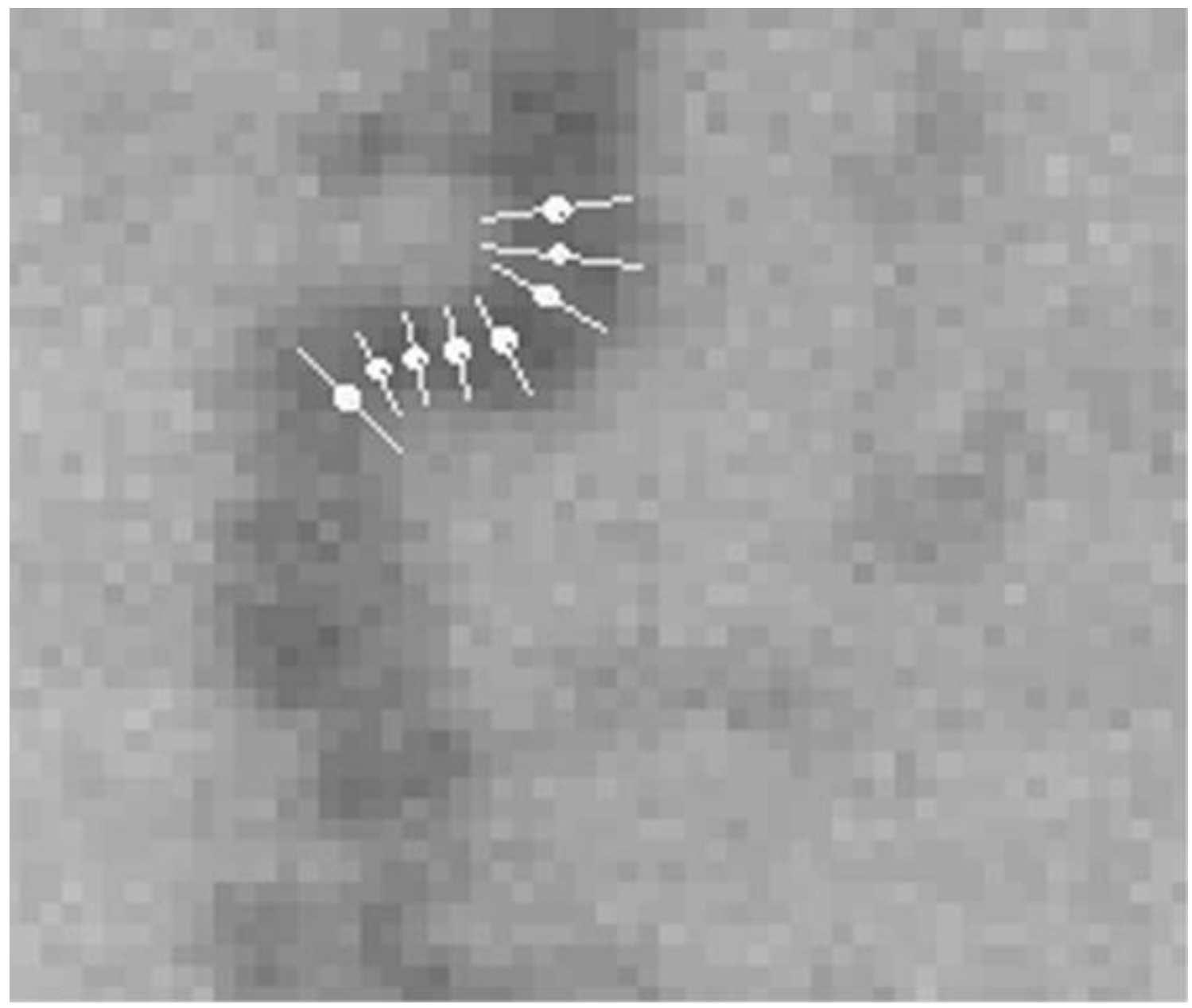

fig9c4.eps 


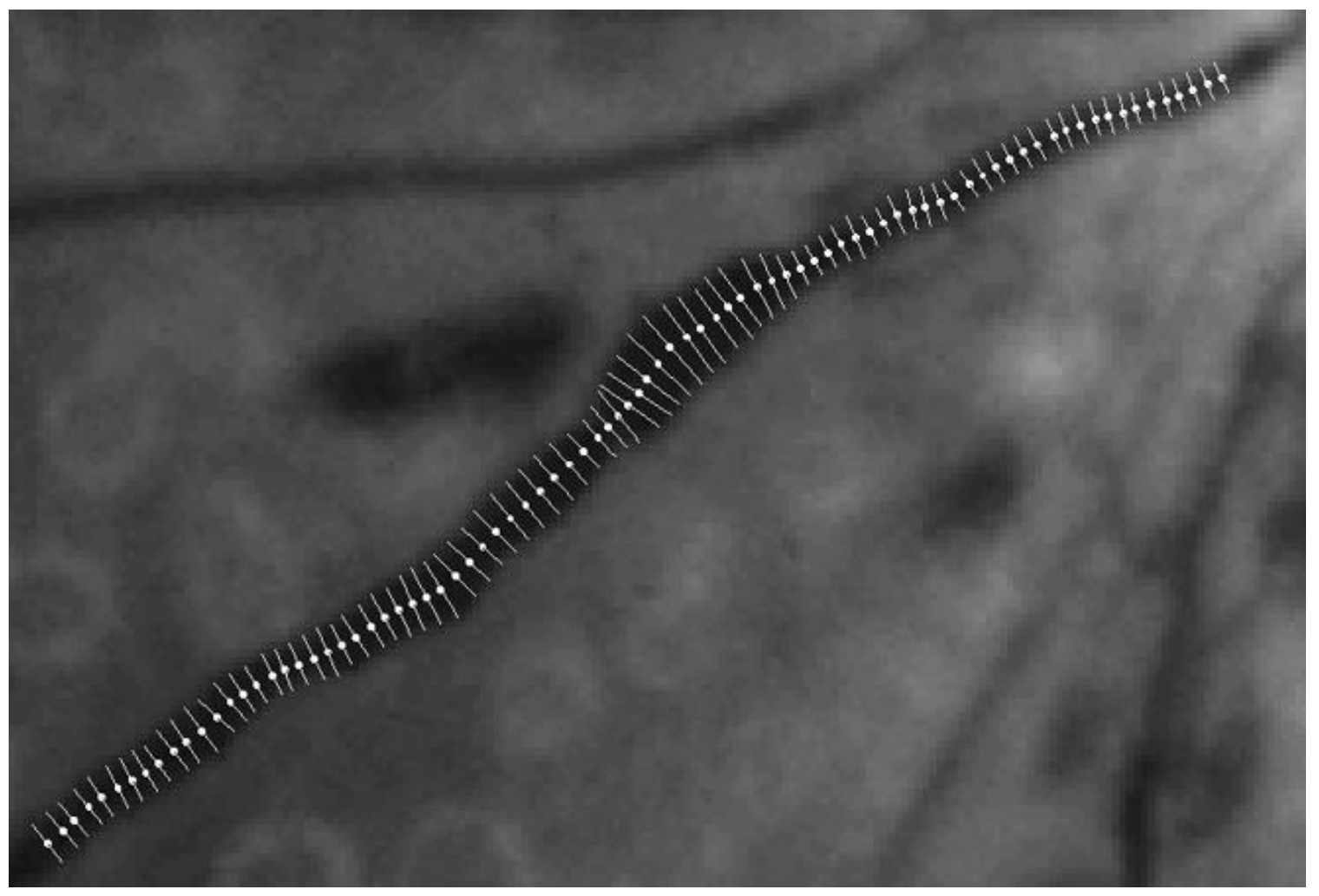

fig9b.eps 


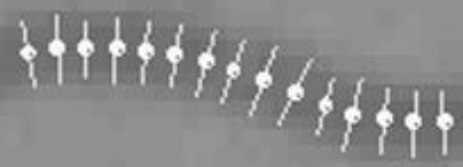

\section{ithititits.}

fig9c.eps 